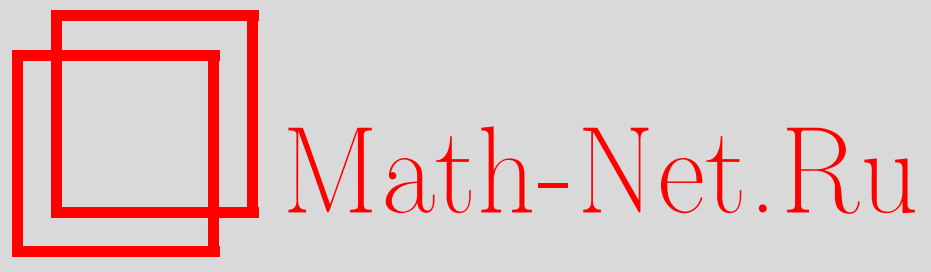

В. П. Платонов, Новые свойства арифметических групп, УМН, 2010, том 65, выпуск 5, 157-184

DOI: https://doi.org/10.4213/rm9374

Использование Общероссийского математического портала Math-Net.Ru подразумевает, что вы прочитали и согласны с пользовательским соглашением http://www . mathnet.ru/rus/agreement

Параметры загрузки:

IP: 54.162 .85 .209

26 апреля 2023 г., $17: 47: 53$

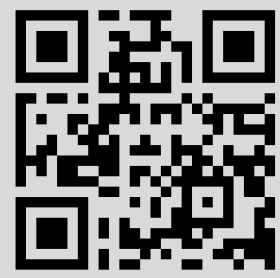




\section{Новые свойства арифметических групп}

\section{В. П. Платонов}

В последние 10-12 лет были получены новые существенные результаты, содержащие решение ряда принципиальных проблем. Были построены первые и довольно неожиданные примеры конечных расширений арифметических групп, не являющихся арифметическими; найден критерий арифметичности подобных расширений; доказаны глубокие теоремы жесткости для арифметических подгрупп алгебраических групп с радикалом; доказана теорема конечности числа классов сопряженности конечных подгрупп в конечных расширениях арифметических групп, имеющая многочисленные приложения, в частности, позволившая решить проблему Бореля-Серра (1964) о конечности первых когомологий конечных групп с коэффициентами в арифметической группе; решена проблема, поставленная более 30 лет назад, о существовании целочисленных линейных групп с конечным числом образующих, имеющих бесконечное число классов сопряженности конечных подгрупп; решена проблема арифметичности для разрешимых групп.

Аналогичные проблемы решены и для решеток в группах Ли с конечным числом связных компонент. В статье дается обзор отмеченных выше результатов.

Библиография: 27 названий.

Ключевые слова: арифметическая группа, теоремы жесткости, критерий арифметичности, проблема сопряженности конечных подгрупп, решетки в группах Ли.

\section{СоДЕРЖАНИЕ}

1. Введение.............................................. 158

2. Конечные расширения арифметических групп................. 158

3. Конечные подгруппы в конечных расширениях арифметических групп 162

4. Целочисленные линейные группы с конечным числом образующих . . . 171

5. Проблема арифметичности для разрешимых групп............... 173

6. Решетки в группах Ли . . . . . . . . . . . . . . . . . . . . . . 179

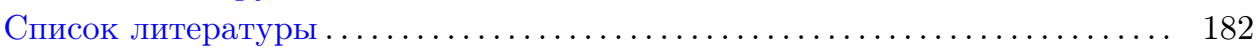

Работа выполнена при поддержке РФФИ (гранты № 09-01-00287, 09-01-12169). 


\section{1. Введение}

Пусть $G \subset G L_{n}(\mathbb{C})$ - линейная алгебраическая группа, определенная над полем $\mathbb{Q}$ рациональных чисел. Подгруппа $H \subset G$ называется арифметической подгруппой $G$, если $H$ соизмерима с $G(\mathbb{Z})=G \cap G L_{n}(\mathbb{Z})$. Напомним, что две подгруппы $H_{1}, H_{2} \subset G L_{n}(\mathbb{C})$ называются соизмеримыми, если их пересечение $H_{1} \cap H_{2}$ имеет конечный индекс в $H_{1}$ и $H_{2}$.

Абстрактная группа $Г$ называется арифметической, если она изоморфна арифметической подгруппе подходящей $\mathbb{Q}$-определенной алгебраической группы.

Теория арифметических групп является одной из глубоких областей современной математики. Основные результаты теории арифметических групп, полученные до 1991 г., изложены в книге [1], а также обзоре [2].

В последние 10-12 лет были получены новые существенные результаты, содержащие решение ряда принципиальных проблем. Были построены первые и довольно неожиданные примеры конечных расширений арифметических групп, не являющихся арифметическими; найден критерий арифметичности подобных расширений; доказаны глубокие теоремы жесткости для арифметических подгрупп алгебраических групп с радикалом; доказана теорема конечности числа классов сопряженности конечных подгрупп в конечных расширениях арифметических групп, имеющая многочисленные приложения, в частности, позволившая решить проблему Бореля-Серра (1964) о конечности первых когомологий конечных групп с коэффициентами в арифметической группе; решена проблема, поставленная более 30 лет назад, о существовании целочисленных линейных групп с конечным числом образующих, имеющих бесконечное число классов сопряженности конечных подгрупп; решена проблема арифметичности для разрешимых групп.

Естественно возникают аналогичные проблемы для решеток в группах Ли. Пусть $G$ - вещественная группа Ли с конечным числом связных компонент. Дискретная подгруппа $\Gamma \subset G$ называется решеткой в $G$, если факторпространство $G / \Gamma$ имеет конечную $G$-инвариантную меру. Соответствующие результаты для решеток в группах Ли получены в [3].

Главной целью настоящей статьи является изложение и обстоятельное обсуждение отмеченных выше результатов.

Большинство из них было получено в цикле работ $Ф$. Груневальда и автора [3]-[8].

\section{2. Конечные расширения арифметических групп}

Естественный базисный вопрос, возникающий здесь с самого начала: будет ли конечное расширение арифметической группы снова арифметической группой? Вопреки общим ожиданиям Груневальд и автор построили примеры конечных расширений арифметических групп, которые (расширения) не являются арифметическими, и доказали общий критерий арифметичности конечных расширений арифметических групп. 
Мы начнем с исторически первого контрпримера, построенного Груневальдом и автором (см. [4], [7]).

Группа $S L_{2}(\mathbb{Z})$ содержит подгруппу конечного индекса, которая является свободной группой $F_{3}$ с тремя свободными образующими.

ТЕОРема 2.1. Пусть $F_{3}$ - свободная группа со свободными образующими $x_{1}, x_{2}, x_{3}$. Определим автоморфизм $\sigma$ группы $F_{3}$ следующим образом: $\sigma\left(x_{3}\right)=$ $x_{3}^{-1}, \sigma\left(x_{1}\right)=x_{1}, \sigma\left(x_{2}\right)=x_{2}$. Пусть $T$ - подгруппа группы автоморфизмов $\operatorname{Aut}\left(F_{3}\right)$, порожденная $\sigma$. Тогда расщепляемое расширение $\Delta=F_{3} \rtimes T$ не является арифметической группой.

ДокАЗАТЕЛЬСтво. Мы будем записывать элементы группы $\Delta$ в виде пар $(f, \alpha)\left(f \in F_{3}, \alpha \in T\right)$ с соответствующим умножением.

Прежде всего покажем, что каждая нетривиальная нормальная подгруппа $N$ группы $\Delta$ пересекается с $F_{3}=\left(F_{3}, 1\right) \subset \Delta$ нетривиально и является бесконечной.

Пусть $1 \neq(f, \alpha)=g$ - какой-то нетривиальный элемент из $N$, где $f \in F_{3}$, $\alpha \in T$. Мы можем предполагать, что $\alpha \neq 1$, и, с помощью сопряжения подходящим элементом из $\Delta$, можем добиться выполнения неравенства $f \neq 1$. Рассмотрим соотношение $(f, \alpha)\left(f^{-1}, 1\right)(f, \alpha)(f, 1)=\left(f^{2}, 1\right) \in N$. Так как $F_{3}$ не имеет кручения, то $\left(f^{2}, 1\right)$ является нетривиальным элементом из $F_{3} \cap N$ бесконечного порядка. Следовательно, всякая неединичная нормальная подгруппа группы $\Delta$ бесконечна.

Предположим, что $\Delta$ - арифметическая группа. Тогда существует такая $\mathbb{Q}$-определенная линейная алгебраическая группа $G$, что $\Delta$ изоморфна арифметической подгруппе группы $G(\mathbb{Q})$, которую мы также обозначаем через $\Delta$. При этом без ограничения общности можно считать, что $\Delta$ плотна в $G(\mathbb{Q})$ в топологии Зариского. Если $R$ - разрешимый радикал группы $G$, то $R \cap \Delta=1$, так как $F_{3} \cap R=1$, ибо $F_{3}$ не имеет неединичных разрешимых нормальных подгрупп. Следовательно, можно считать, что связная компонента единицы $G^{\circ}$ является полупростой группой присоединенного типа. Тогда $G^{\circ}=G_{1} \times \cdots \times G_{k}$, где группы $G_{i}, i=1, \ldots, k$, являются $\mathbb{Q}$-простыми линейными алгебраическими группами, определенными над $\mathbb{Q}$.

Теперь мы покажем, что группу $G^{\circ}$ можно считать $\mathbb{Q}$-простой. Пусть $\Gamma_{i}=$ $F_{3} \cap G_{i}$. Заметим, что $\Gamma_{i}$ централизует $\Gamma_{j}$ для $i \neq j$. Так как $F_{3} \cap G^{\circ}$ является арифметической подгруппой группы $G^{\circ}$, то группа $\Gamma_{1} \cdot \Gamma_{2} \cdots \Gamma_{k}$ имеет конечный индекс в $F_{3} \cap G^{\circ}$. Так как никакая подгруппа конечного индекса в $F_{3}$ не содержит нетривиальную пару взаимно централизующих нормальных подгрупп, то существует такой индекс $i_{0} \in 1, \ldots, k$, что $F_{3} \cap G_{i_{0}}$ имеет конечный индекс в $F_{3} \cap G^{\circ}$ и $F_{3} \cap G_{i}=1$ для $i \neq i_{0}$.

Так как $F_{3}$ не имеет кручения, то это влечет, что $F_{3} \subset G_{i_{0}}$. Если учесть, что $F_{3}$ нормальна в $\Delta$ и $\Delta$ плотна в $G$ в топологии Зариского, мы получаем, что $G$ нормализует $G_{i_{0}}$. Остается заметить, что образ $\Delta$ в $G / G_{i_{0}}$ является плотным, поэтому факторгруппа $G / G_{i_{0}}$ конечна. Это доказывает, что группу $G^{\circ}$ можно считать $\mathbb{Q}$-простой. 
Итак, группа $G^{\circ}$ является $\mathbb{Q}$-определенной $\mathbb{Q}$-простой линейной алгебраической группой. Отсюда следует, что $\Delta$ является неприводимой решеткой в группе $G(\mathbb{R})$.

Следуя Ивасаве, мы введем характеристический индекс $\chi(H)$ вещественной группы Ли $H$ с конечным числом связных компонент: $\chi(H)=\operatorname{dim}_{\mathbb{R}}(H)-$ $\operatorname{dim}_{\mathbb{R}}(B)$, где $\operatorname{dim}_{\mathbb{R}}(H)$ есть размерность $H$ и $B$ - максимальная компактная подгруппа в $H$. Так как $F_{3}$ изоморфна подгруппе конечного индекса в $P S L_{2}(\mathbb{Z}) \subset$ $P S L_{2}(\mathbb{R})$, то из результата работы [9] следует, что $\chi(G(\mathbb{R})) \leqslant \chi\left(P S L_{2}(\mathbb{R})\right)=2$. Отсюда следует, что $G(\mathbb{R})$ как вещественная группа Ли разлагается следующим образом: $G(\mathbb{R}) \cong H_{1} \times H_{2}$, где $H_{1}$ является конечным расширением группы $P S L_{2}(\mathbb{R})$, а фактор $H_{2}$ является компактной группой Ли. Пересечение $\Delta \cap H_{2}$ является конечной нормальной подгруппой. Это означает, что $\Delta \cap H_{2}=1$, так как нормальная подгруппа $\Delta$ может быть либо тривиальной, либо бесконечной.

Следовательно, мы можем рассматривать $\Delta$ (посредством проекции) как подгруппу группы $H_{1}$. Пусть $H_{1}^{\circ}$ - связная компонента единицы в $H_{1}$. По построению, $H_{1}^{\circ}$ является вещественной группой Ли, изоморфной группе $P S L_{2}(\mathbb{R})$. Положим $N=F_{3} \cap H_{1}^{\circ}$. Тогда $N$ является нормальной подгруппой конечного индекса в $\Delta$. Выберем $n \in N$ таким образом, что $x_{1}^{n}, x_{2}^{n}, x_{3}^{n} \in N$. Сопряжение посредством $(\sigma, 1)$ индуцирует аналитический автоморфизм $\tilde{\delta}$ группы $H_{1}^{\circ}$. Так как все автоморфизмы группы $P S L_{2}(\mathbb{C})$ внутренние, то существует $\gamma \in P S L_{2}(\mathbb{C})$ такой, что $\tilde{\delta}(x)=\gamma x \gamma^{-1}$ для всех $x \in P S L_{2}(\mathbb{R})$.

С точностью до сопряженности,

$$
x_{1}^{n}=\left(\begin{array}{cc}
t & 0 \\
0 & t^{-1}
\end{array}\right) \quad \text { или } \quad x_{1}^{n}=\left(\begin{array}{ll}
1 & 1 \\
0 & 1
\end{array}\right) .
$$

Пусть мы имеем первый случай. Так как порядок элемента $x_{1}^{n}$ бесконечен, то $t$ не может быть корнем из единицы. Тогда $\gamma=\left(\begin{array}{cc}s & 0 \\ 0 & s^{-1}\end{array}\right)$. Из равенства $\gamma x_{n} \gamma^{-1}=x_{n}^{-1}$ следует, что $s \neq \pm 1$. Тогда $\gamma x_{2}^{n} \gamma^{-1}=x_{2}^{n}$ влечет, что элемент $x_{2}^{n}$ является диагональным. А это невозможно. Второй случай рассматривается аналогично. Теорема доказана.

Так как $\left[\Delta / F_{3}\right]=2$, то этот контрпример является минимальным.

Приведем еще один контрпример из [3].

ПРЕДЛОЖЕНИЕ 2.1. Пусть $F_{2}=\left\langle h_{1}, h_{2}\right\rangle$ - свободная группа с двумя образующими. Рассмотрим следующие три автоморфизма группъ $F_{2}$ :

$$
\sigma_{1}\left(h_{1}\right)=h_{1}^{-1}, \quad \sigma_{2}\left(h_{2}\right)=h_{2}^{-1}, \quad \sigma_{3}\left(h_{1}\right)=h_{2}, \quad \sigma_{3}\left(h_{2}\right)=h_{1},
$$

с условием, что $\sigma_{i}$ действует тождественно на образующих, которые не указанъь. Пусть $A$ - подгруппа группь $\operatorname{Aut}\left(F_{2}\right)$, порожденная $\sigma_{1}, \sigma_{2}, \sigma_{3}, u \Delta=$ $F_{2} \rtimes A$. Тогда $\Delta$ не является арифметической группой.

Доказательство предложения 2.1 аналогично доказательству теоремы 2.1 и будет приведено в разделе 6 как часть более общего результата, сформулированного ниже.

Отметим, что $\Delta / F_{2}$ изоморфна диэдральной группе порядка 8 и $F_{2}$ изоморфна подгруппе конечного индекса в $P S L_{2}(\mathbb{Z})$. 
Груневальд и автор в [3] получили полное описание арифметических подгрупп группы $P S L_{2}(\mathbb{R})$, для которых существуют конечные расширения, не являющиеся арифметическими.

ТеОРема 2.2. Если Г - арифметическая подгруппа в $P S L_{2}(\mathbb{R})$, то существует конечное расширение $Г$, не являющееся арифметической группой, в том и только том случае, когда факторпространство $P S L_{2}(\mathbb{R}) / \Gamma$ не является компактным.

Доказательство теоремы 2.2 также будет получено в разделе 6 как часть соответствующего результата для решеток в группах Ли.

Следующая общая теорема показывает, что приведенные выше контрпримеры в определенном смысле являются единственно возможными.

Теорема 2.3. Пусть Г - арифметическая подгруппа в $\mathbb{Q}$-определенной линейной алгебрачческой группе $G$. Предположим, что связная компонента единицы $G(R)^{\circ}$ вещественной группь Ли $G(\mathbb{R})$ не имеет такого простого фактора, изоморфного $P S L_{2}(\mathbb{R})$, что проекция группы $\Gamma \cap G(\mathbb{R})^{\circ}$ в этот фактор является дискретной подгруппой. Тогда всякое конечное расширение группы $Г$ арифметично.

СлеДСТвИЕ. Пусть Г - разрешимая арифметическая группа. Тогда всякое конечное расширение группы Г является арифметической группой.

Доказательство теоремы 2.3 является трудным и длинным и не будет представлено здесь. Оно базируется на новых результатах о жесткости для арифметических подгрупп алгебраических групп с радикалом. Эти новые результаты о жесткости имеют самостоятельный интерес. Мы отсылаем к оригинальной статье [7] за полным изложением доказательства теоремы 2.3. В действительности роль результатов о жесткости достаточно очевидна, если рассмотреть соответствующее индуцированное представление. Например, если арифметическая группа Г обладает свойством сильной жесткости, т. е. всякий автоморфизм $Г$ рационален, то любое конечное расширение $Г$ арифметично. Более детально эта ситуация будет обсуждена в разделе 5.

Мы ограничимся здесь лишь формулировкой одной из теорем жесткости из [7]. Пусть $G_{1}, G_{2}$ - линейные алгебраические группы, определенные над $\mathbb{Q}$, и $\Gamma_{1} \subset G_{1}, \Gamma_{2} \subset G_{2}$ - арифметические подгруппы.

При изучении проблемы жесткости обычно имеют дело с вопросом: когда изоморфизм между арифметическими группами $\Gamma_{1}$ и $\Gamma_{2}$ может быть расширен до рационального (или почти рационального) изоморфизма между алгебраическими группами $G_{1}$ и $G_{2}$ ?

Для не полупростых групп нетрудно построить примеры, когда нет никакой эффективной связи между изоморфизмами арифметических групп и рациональными изоморфизмами содержащих их алгебраических групп (см. [7]).

Тем не менее, довольно общие результаты жесткости для алгебраических групп с радикалом могут быть получены. Всякая $\mathbb{Q}$-определенная линейная алгебраическая группа $G$ может быть представлена в виде $G=H \cdot U(G)$, где $U(G)$ - унипотентный радикал группы $G$, а $H$ - редуктивная группа. Мы 
будем называть группу $G$ группой с сильным унипотентным радикалом, если естественное действие группы $H$ на $U(G)$ сопряжениями является точным. Эквивалентное определение: $G$ называется группой с сильным унипотентным радикалом, если ядро естественного действия группы $G$ на $U(G)$ сопряжениями содержится в $U(G)$.

Теорема 2.4. Пусть $G_{1}, G_{2}$ - $\mathbb{Q}$-определенные линейные алгебраические группы с сильными унипотентными радикалами, и пусть $Z\left(U\left(G_{2}\right)\right)$ - иентр группы $U\left(G_{2}\right)$. Если $\Gamma_{1} \subset G_{1}(\mathbb{Q}), \Gamma_{2} \subset G_{2}(\mathbb{Q})$ - арифметические подгруппъ, плотные в топологии Зариского и $\varphi: \Gamma_{1} \rightarrow \Gamma_{2}-$ изоморфизм, то существуют $\mathbb{Q}$-определенный рациональный изоморфизм $\Phi: G_{1} \rightarrow G_{2}$ и отображение $v: \Gamma_{1} \rightarrow Z\left(U\left(G_{2}\right)\right)$ такие, что $\Phi(\gamma)=v(\gamma) \varphi(\gamma)$ для всех $\gamma \in \Gamma_{1}$, причем $v(\gamma)=1$ для всех $\gamma \in \Gamma_{1} \cap U\left(G_{1}\right)$.

Из теоремы 2.4 следует, что если $Г$ - арифметическая подгруппа $\mathbb{Q}$-определенной линейной алгебраической группы с сильным унипотентным радикалом, то любое конечное расширение Г арифметично.

\section{3. Конечные подгруппы в конечных расширениях арифметических групп}

Основной целью настоящего раздела является доказательство следующей теоремы, имеющей многочисленные применения.

ТЕОрема 3.1. Любое конечное расширение арифметической группы имеет конечное число сопряженных классов конечных подгрупп.

Для доказательства теоремы 3.1 нам понадобятся некоторые дополнительные сведения. Начнем с определений и обозначений.

Пусть $n \in \mathbb{N}$ - натуральное число, а $S_{n}$ - симметрическая группа перестановок множества $\{1, \ldots, n\}$. Для множества $X$ обозначим через $X^{[n]}=X \times \cdots \times X$ его произведение в количестве $n$-экземпляров. Для группы Г обозначим через Г $S_{n}$ ее сплетение. А именно:

$$
\Gamma \curlywedge S_{n}=\left\{\left(\gamma_{1}, \ldots, \gamma_{n} ; \pi\right) \mid \gamma_{1}, \ldots, \gamma_{n} \in \Gamma, \pi \in S_{n}\right\}
$$

Умножение в сплетении определяется следующей формулой:

$$
\left(\gamma_{1}, \ldots, \gamma_{n} ; \pi\right)\left(\gamma_{1}^{\prime}, \ldots, \gamma_{n}^{\prime} ; \pi^{\prime}\right)=\left(\gamma_{1} \gamma_{\pi^{-1}(1)}^{\prime}, \ldots, \gamma_{n} \gamma_{\pi^{-1}(n)}^{\prime} ; \pi \pi^{\prime}\right)
$$

где $\gamma_{1}, \ldots, \gamma_{n}, \gamma_{1}^{\prime}, \ldots, \gamma_{n}^{\prime} \in \Gamma$ и $\pi, \pi^{\prime} \in S_{n}$. Имеем гомоморфизм

$$
\Phi: \Gamma \imath S_{n} \rightarrow S_{n}, \quad \Phi:\left(\gamma_{1}, \ldots, \gamma_{n} ; \pi\right) \mapsto \pi .
$$

Для подгруппы $G \subseteq \Gamma$ २ $S_{n}$ и числа $i \in\{1, \ldots, n\}$ определим

$$
G_{\{i\}}=\{g \in G \mid \Phi(g)(i)=i\} .
$$

Назовем подгруппу $G \subseteq \Gamma$ ? $S_{n}$ транзитивной, если $\Phi(G)$ действует транзитивно на множестве $\{1, \ldots, n\}$. Пусть $\Gamma-$ подгруппа, a $\operatorname{Aut}(\Gamma)-$ группа ее 
автоморфизмов. Отождествим сплетение $\operatorname{Aut}(\Gamma)$ 乙 $S_{n}$ с подгруппой в $\operatorname{Aut}\left(\Gamma^{[n]}\right)$ следующим образом:

$$
\left(\varphi_{1}, \ldots, \varphi_{n} ; \pi\right)\left(\gamma_{1}, \ldots, \gamma_{n}\right)=\left(\varphi_{1}\left(\gamma_{\pi^{-1}(1)}\right), \ldots, \varphi_{n}\left(\gamma_{\pi^{-1}(n)}\right)\right)
$$

для $\varphi_{1}, \ldots, \varphi_{n} \in \operatorname{Aut}(\Gamma), \pi \in S_{n}$ и $\gamma_{1}, \ldots, \gamma_{n} \in \Gamma$. Тем самым для подгруппы $G \subseteq \operatorname{Aut}(\Gamma)$ 乙 $S_{n}$ мы можем определить расщепимое расширение $\Gamma^{[n]} \rtimes G$. Обозначим через

$$
\Gamma_{i}=\left\{\left(\gamma_{1}, \ldots, \gamma_{n}\right) \in \Gamma^{[n]} \mid \gamma_{j}=1 \text { для всех } j \neq i\right\}
$$

$i$-й множитель в прямом произведении. Подгруппа $G_{\{i\}}$ нормализует $\Gamma_{i}$ и $\Gamma_{i} \rtimes G_{\{i\}}$ вкладывается в качестве подгруппы в $\Gamma^{[n]} \rtimes G$.

Лемма 3.1. Пусть $n \in \mathbb{N}$ - натуральное число, и пусть $G_{1}, \ldots, G_{n}-\mathbb{Q}$-простые линейные алгебраические группы, определенные над $\mathbb{Q}$. Предположим, что ни одна из групп $G_{i}(\mathbb{R})$ не является компактной. Положим $G=G_{1} \times$ $\cdots \times G_{n}$. Пусть $\Gamma \subseteq G(\mathbb{Q})$ - арифметическая подгруппа, а $\varphi: \Gamma \rightarrow \Gamma-e е$ группа автоморфизмов. Также положим $\Gamma_{i}=G_{i} \cap \Gamma$ для $i=1, \ldots, n$. Тогда найдется перестановка $\pi$ множества $\{1, \ldots, n\}$ такая, что $\varphi\left(\Gamma_{i}\right)=\Gamma_{\pi(i)} \partial л я$ $i=1, \ldots, n$.

ДоказАтельство. Пусть $\widetilde{\Gamma}=\Gamma_{1} \cdots \Gamma_{n}$. Группа $\widetilde{\Gamma}$ имеет конечный индекс в Г и тем самым является арифметической. Кроме того, $\varphi(\widetilde{\Gamma})-$ подгруппа конечного индекса в Г и, следовательно, арифметична. Для $i \in\{1, \ldots, n\}$ определим

$$
\Gamma^{i}=\Gamma_{1} \cdots \Gamma_{i-1} \cdot \Gamma_{i+1} \cdots \Gamma_{n}
$$

как произведение всех групп $\Gamma_{j}$ кроме $\Gamma_{i}$. Группы $\Gamma_{i}$ и $\Gamma^{i}$ являются централизаторами друг друга. Для $j \in\{1, \ldots, n\}$ определим

$$
\operatorname{pr}_{j}: G=G_{1} \times \cdots \times G_{n} \rightarrow G_{j}
$$

как проекцию на $j$-й множитель. Для каждого $i \in\{1, \ldots, n\}$ существует по крайней мере одно $j \in\{1, \ldots, n\}$ такое, что $\operatorname{pr}_{j}\left(\varphi\left(\Gamma_{i}\right)\right) \neq\{1\}$. В этом случае мы покажем, что $\operatorname{pr}_{j}\left(\varphi\left(\Gamma^{i}\right)\right)=\{1\}$. Пусть $H_{1}=\overline{\operatorname{pr}_{j}\left(\varphi\left(\Gamma_{i}\right)\right)}, H_{2}=\overline{\operatorname{pr}_{j}\left(\varphi\left(\Gamma^{i}\right)\right)}-$ соответствующие замыкания рассматриваемых подгрупп в $G_{j}$ в топологии Зариского. Это две подгруппы в $G_{j}$, определенные над $\mathbb{Q}$. Их произведение $H_{1} \cdot H_{2}$ - также замкнутая подгруппа, определенная над $\mathbb{Q}$, поскольку $H_{1}$ централизует $H_{2}$. Более того, по теореме плотности Бореля подгруппа $H_{1} \cdot H_{2}$ плотна в топологии Зариского в $G_{j}$, поскольку она содержит арифметическую подгруппу $\operatorname{pr}_{j}\left(\varphi\left(\Gamma_{i}\right)\right) \cdot \operatorname{pr}_{j}\left(\varphi\left(\Gamma^{i}\right)\right)=\operatorname{pr}_{j}(\varphi(\widetilde{\Gamma}))$, плотную в топологии Зариского в $G_{j}$. Здесь мы воспользовались тем, что ни одна из групп $G_{i}(\mathbb{R})$ не является компактной. Итак, имеем $H_{1} \cdot H_{2}=G_{j}$. В частности, $H_{1}$ - определенная над $\mathbb{Q}$ нормальная подгруппа в $G_{j}$. Поскольку $H_{1} \neq\{1\}$, мы получаем, что $H_{1}=G_{j}$. Если $H_{2} \neq\{1\}$, то $H_{2}=G_{j}$ и группа $G_{j}$ коммутативна, что приводит нас к противоречию.

Мы доказали, что для каждого $i \in\{1, \ldots, n\}$ существует $j \in\{1, \ldots, n\}$ такое, что $\operatorname{pr}_{j}\left(\varphi\left(\Gamma_{i}\right)\right) \neq\{1\}$ и $\operatorname{pr}_{j}\left(\varphi\left(\Gamma_{l}\right)\right)=\{1\}$ для каждого $l \neq i$. Тем самым 
$j=\pi(i)$ однозначно определяется по $i$, и, кроме того, $\varphi\left(\Gamma_{i}\right) \subseteq \Gamma_{\pi(i)}$. Применяя предыдущее рассуждение к $\varphi^{-1}$, мы получаем, что $\varphi\left(\Gamma_{i}\right)=\Gamma_{\pi(i)}$. Лемма доказана.

В качестве прямого следствия леммы 3.1 мы получаем следующее предложение.

ПРЕДЛОЖЕНИЕ 3.1. Пусть $G$ - линейная алгебраическая группа, определенная над $\mathbb{Q}$, которая является простой над $\mathbb{Q}$. И пусть $\Gamma \subseteq G(\mathbb{Q})$ - бесконечная арифметическая подгруппа. Тогда всех $n \in \mathbb{N}$

$$
\operatorname{Aut}\left(\Gamma^{[n]}\right)=\operatorname{Aut}(\Gamma) \imath S_{n} .
$$

Заметим, что предложение 3.1 можно применить, когда Г - свободная группа ранга, строго большего единицы. В этом случае Г может быть вложена в качестве подгруппы конечного индекса в $P S L_{2}(\mathbb{Z}) \subseteq P S L_{2}(\mathbb{Q})$. (Под рангом $\operatorname{rk}(\Gamma)$ свободной группы $Г$ мы понимаем количество элементов в базисе группы $\Gamma)$.

Для краткости изложения группу $\Delta$ мы будем называть $f c f$-груnпой, если она содержит только конечное число классов сопряженности конечных подгрупп.

Пусть $(X, d)$ - метрическое пространство, и пусть $G$ - группа, действующая на $X$ изометриями. Мы говорим, что $G$ действует кокомпактно на $X$, если факторпространство $G \backslash X$ - компактно. Если же для каждого компактного подмножества $K \subset X$ существует лишь конечное число элементов $g \in G$ таких, что $K \cap g K \neq \varnothing$, то мы говорим, что $G$ действует разрывно на $X$. Следующая лемма, несмотря на свою очевидность, является базисным утверждением для установления свойства группы являться $f c f$-группой.

Лемма 3.2. Пусть $(X, d)$ - метрическое пространство, и пусть $\Delta$ - группа изометрий $X$, действующая разрывно и кокомпактно на $X$. Предположим, что каждая конечная подгруппа изометрий $X$ имеет неподвижную точку. Тогда $\Delta$ является $f$ сf-группой.

Примером метрических пространств, для которых любая конечная подгруппа группы изометрий имеет неподвижную точку, являются римановы многообразия строго отрицательной кривизны (например, $S L_{2}(\mathbb{R}) / S O_{2}(\mathbb{R})$ ) или, более общо, САТ(0)-пространства. Это, например, доказано [10]. Если $(X, d)$ - метрическое пространство, то мы всегда считаем, что метрика на $X^{[n]}$ задана формулой

$$
d^{[n]}\left(\left(x_{1}, \ldots, x_{n}\right),\left(y_{1}, \ldots, y_{n}\right)\right)=\sqrt{d\left(x_{1}, y_{1}\right)^{2}+\cdots+d\left(x_{n}, y_{n}\right)^{2}} .
$$

Следующий важный технический результат понадобится нам в доказательстве теоремы 3.1 .

ЛЕмма 3.3. Для $n \in \mathbb{N}$ и группы $\Gamma$ рассмотрим транзитивную подгруппу $G \subseteq \operatorname{Aut}(\Gamma)$ 乙 $S_{n}$. Пусть $G_{\{1\}}, \Gamma_{1}$ определены по формулам (3.1) и (3.2) соответственно. Предположим, что $(X, d)$ - метрическое пространство, на котором $\Gamma_{1} \rtimes G_{\{1\}}$ действует изометриями. Тогда существует изометричное действие $\Gamma^{[n]} \rtimes G$ на $\left(X^{[n]}, d^{[n]}\right)$ такое, что первая проекция $p_{1}: X^{[n]} \rightarrow X$ эквивариантна относительно $\Gamma_{1} \rtimes G_{\{1\}}$ и выполнены следующие условия: 
(i) если $\Gamma_{1} \rtimes G_{\{1\}}$ действует кокомпактно на $X$, то также действует $\Gamma^{[n]} \rtimes G$ на $X^{[n]}$;

(ii) если $\Gamma_{1} \rtimes G_{\{1\}}$ действует разрьвно на $X$, то также действует $и \Gamma^{[n]} \rtimes G$ нa $X^{[n]}$.

ДокАЗАТЕЛЬСтво. Выберем представителей правых смежных классов $r_{1}, \ldots, r_{n}$ группы $G_{\{1\}}$ в $G$ такие, что $\Phi\left(r_{i}\right)(1)=i$ для $i=1, \ldots, n$ и $r_{1}=1$. Имеем изоморфизмы

$$
\psi_{i}: \Gamma_{i} \rtimes G_{\{i\}} \rightarrow \Gamma_{1} \rtimes G_{\{1\}}, \quad \psi_{i}(h)=r_{i}^{-1} h r_{i} \quad(i=1, \ldots, n) .
$$

Мы воспользуемся ими, чтобы определить действие группы $\Gamma^{[n]}$ на $X^{[n]}$ формулой

$$
\left(\gamma_{1}, \ldots, \gamma_{n}\right) \cdot\left(x_{1}, \ldots, x_{n}\right)=\left(\psi_{1}\left(\gamma_{1}\right) \cdot x_{1}, \ldots, \psi_{n}\left(\gamma_{n}\right) \cdot x_{n}\right)
$$

Опишем теперь действие группы $G$ на $X^{[n]}$. Для $g \in G$ и $i=1, \ldots, n$ имеем

$$
u(g, i)=r_{\Phi(g)(i)}^{-1} \cdot g \cdot r_{i} \in G_{\{1\}} .
$$

Заметим, что $u\left(g g^{\prime}, i\right)=u\left(g, \Phi\left(g^{\prime}\right)(i)\right) \cdot u\left(g^{\prime}, i\right)$ и $u\left(g^{-1}, i\right)=u\left(g, \Phi\left(g^{-1}\right)(i)\right)^{-1}$. Теперь мы можем определить действие $g \in G$ на $x_{1}, \ldots, x_{n} \in X^{[n]}$ формулой

$$
g \cdot\left(x_{1}, \ldots, x_{n}\right)=\left(u\left(g, \Phi(g)^{-1}(1)\right) \cdot x_{\Phi(g)^{-1}(1)}, \ldots, u\left(g, \Phi(g)^{-1}(n)\right) \cdot x_{\Phi(g)^{-1}(n)}\right) .
$$

Непосредственные вычисления показывают, что формулы (3.3) и (3.4) согласованы и определяют изометрическое действие $\Gamma^{[n]} \rtimes G$ на $\left(X^{[n]}, d^{[n]}\right)$. Также несложно проверить свойства (i) и (ii). Лемма доказана.

В наших рассуждениях нам часто будет полезно следующее наблюдение.

Лемма 3.4. Пусть $1 \rightarrow N \rightarrow G \rightarrow H \rightarrow 1$ - точная последовательность групп. Предположим, что всякое конечное расширение группы $N$ является $f c f$-группой, а также что $H$ является $f c f$-группой. Тогда $G$ - $f c f$-группа.

ДокАзАтельство. Поскольку $H-f c f$-группа, нам надо показать, что конечные подгруппы $G$, которые лежат в фиксированном конечном расширении $N$ в $G$, распадаются в конечное число классов сопряженности. Но это мы потребовали в условии леммы.

Мы назовем расширение группы $\Gamma \subseteq \Delta$ нормальным, если $\Gamma$ - нормальная подгруппа в $\Delta$. Приведем следующее простое, но полезное наблюдение.

Лемма 3.5. Пусть Г - группа без кручения. Предположим, что всякое расщепимое расширение Г с помощью конечной группы является $f c f$-группой. Тогда любое конечное нормалъное расширение группы Г обладает этим свойством.

ДоказАТЕЛьство. Пусть $\Delta-$ конечное нормальное расширение Г. Выберем конечные подгруппы $\Gamma_{1}, \ldots, \Gamma_{s} \subseteq \Delta$ такие, что любая подгруппа (конечной) факторгруппы $\Delta / \Gamma$, которая является образом при морфизме факторизации конечной подгруппы в $\Delta$, является образом одной из групп $\Gamma_{1}, \ldots, \Gamma_{s}$. 
Заметим, что морфизм факторизации $\Delta \rightarrow \Delta / \Gamma$ инъективен на каждой конечной подгруппе $\Delta$. Положим $\Delta_{i}=\left\langle\Gamma, \Gamma_{i}\right\rangle$ для $i=1, \ldots, s$. Все $\Delta_{i}$ являются расщепимыми расширениями группы $\Gamma$. В силу выбора $\Gamma_{1}, \ldots, \Gamma_{s}$ каждая конечная подгруппа в $\Delta$ содержится в одной из подгрупп $\Delta_{i}$. Откуда следует наш результат.

Прежде чем привести доказательство теоремы 3.1, обсудим некоторые ее частные случаи. Первый - когда Г является прямым произведением арифметических подгрупп в $P S L_{2}(\mathbb{C})$, которые не являются кокомпактными в $P S L_{2}(\mathbb{R})$.

ПРЕДЛОЖЕНИЕ 3.2. Пусть $Г=F_{1} \times \cdots \times F_{n}-$ прямое произведение конечно порожденных свободных групп $F_{i} c \operatorname{rk}\left(F_{i}\right) \geqslant 2(i=1, \ldots, n)$. Пусть $\Delta-$ конечное расширение группъ $\Gamma$. Тогда $\Delta$ является $f c f$-группой.

ДокАЗАТЕЛЬство. Начнем с изучения случая $n=1$. В этом случае $\Delta$ является конечным расширением конечно порожденной свободной группы. Согласно конструкции из книги [12; гл. 3], существует дерево $T(\Delta)$, снабженное метрикой, такое, что $\Delta$ точно действует на нем изометриями $T(\Delta)$. Это действие разрывно, что влечет конечность стабилизаторов его вершин. Более того, фактор $\Delta \backslash T(\Delta)$ компактен. Пусть $\Gamma$ - конечная подгруппа в $\Delta$. По теореме о неподвижной точке (см. [11; гл. 4]) группа Г имеет неподвижную точку на $T(\Delta)$. Сопрягая $\Gamma$ с помощью элемента из $\Delta$, мы можем предположить, что Г оставляет неподвижными только одну из конечного числа вершин дерева $T(\Delta)$. Перейдем к случаю, когда $n>1$. Поскольку Г имеет конечный индекс в $\Delta$, существует подгруппа $N$ группы $\Gamma$, которая нормальна и имеет конечный индекс в $\Delta$. Определим $\Gamma_{i}=N \cap F_{i}$ для $i=1, \ldots, n$, а также $N_{0}=\Gamma_{1} \times \cdots \times \Gamma_{n}$. Здесь мы снова отождествили $F_{i}$ с соответствующим множителем в $\Gamma$. Тогда $\Gamma_{i}$ - подгруппа конечного индекса в $F_{i}$ и, следовательно, является свободной подгруппой ранга $\operatorname{rk}\left(\Gamma_{i}\right) \geqslant 2$. Заметим также, что $N_{0}$ имеет конечный индекс в $\Delta$. Вложим каждую группу $\Gamma_{i}$ в качестве подгруппы конечного индекса в $P S L_{2}(\mathbb{Z})$. Мы отождествим каждую группу $F_{i}$ с ее вложением в качестве арифметической подгруппы в $P S L_{2}(\mathbb{C})$. По лемме 3.1 мы получаем, что $N_{0}$ нормальна в $\Delta$. Чтобы доказать предложение, мы можем считать, используя лемму 3.5 , что $\Delta$ является расщепимым расширением $\Delta \cong N_{0} \rtimes \mathrm{g}$ для некоторой конечной подгруппы $\mathbf{g}$.

Теперь перенумеруем $\Gamma_{1}, \ldots, \Gamma_{n}$ таким образом, что $\Gamma_{1}, \ldots, \Gamma_{k}$ изоморфны группе $\Gamma_{1}$, а $\Gamma_{k+1}, \ldots, \Gamma_{n}$ ей не изоморфны. Положим $N_{1}=\Gamma_{1} \cdots \Gamma_{k}$ и $N_{2}=$ $\Gamma_{k+1} \cdots \Gamma_{n}$. По лемме 3.1 обе группы $N_{1}$ и $N_{2}$ нормальны в $\Delta$. Вкладывая g диагонально в $\mathrm{g} \times \mathrm{g}$, мы получаем, что $\Delta$ изоморфна подгруппе конечного индекса в $\left(N_{1} \rtimes \mathbf{g}\right) \times\left(N_{2} \rtimes \mathbf{g}\right)$.

Теперь рассмотрим группу $\Delta_{1}=N_{1} \rtimes \mathrm{g}$. Мы воспользуемся терминологией, введенной в начале раздела. Факторизуя группу $\mathbf{g}$ по централизатору $N_{1}$ в $\mathbf{g}$, мы можем считать, что $\mathbf{g}$ - подгруппа $\operatorname{Aut}(\Gamma)$ 乙 $S_{k}$.

Предположим вначале, что $\mathbf{g}$ транзитивна на $\{1, \ldots, k\}$. Рассмотрим тогда группу $\Gamma_{1} \rtimes \mathbf{g}_{\{1\}}$ (обозначения см. в (3.1), (3.2)). Это конечное расширение конечно порожденной свободной группы, и мы можем построить снабженное метрикой дерево $T=T\left(\Gamma_{1} \rtimes \mathbf{g}_{\{1\}}\right)$, упоминавшееся выше. Дерево $T$ является 
CAT(0)-пространством (см. [10]), и таким же пространством является прямое произведение $T^{[k]}$ (см. [13]).

По лемме 3.3 группа $\Gamma_{1} \rtimes \mathbf{g}_{\{1\}}$ действует изометриями разрывно и кокомпактно на $T^{[k]}$. Поскольку конечная группа изометрий $\mathrm{CAT}(0)$-пространства имеет неподвижную точку (см. [10]), лемма 3.2 утверждает, что $N_{1} \rtimes \mathbf{g}$ является $f c f$-группой в случае, когда $\mathbf{g}$ транзитивна.

Общий случай следует из леммы 3.4 с помощью индукции по числу орбит $\mathbf{g}$ в $\{1, \ldots, k\}$. Предложение доказано.

Утверждение, аналогичное предложению 3.2 , для конечных расширений свободных групп бесконечного ранга неверно. Минимальный контрпример (т. е. когда $\left.\left[\Delta: F_{\infty}\right]=2\right)$ приведен в статье [14].

ПрЕДЛОЖЕНИЕ 3.3. Пусть $\Gamma \subset P S L_{2}(\mathbb{R})$ - кокомпактная решетка, и пусть $\Delta \subseteq \Gamma$ - конечное расширение Г. Тогда выполнено следующее.

(i) Если Г - группа без кручения, нормальная в $\Delta$, mо $\Delta / Z_{\Delta}(\Gamma)$ изоморфна кокомпактной дискретной подгруппе в $P S L_{2}(\mathbb{R})$.

(ii) $B$ общем случае существует конечное расширение $H$ группъ $P S L_{2}(\mathbb{R})$ такое, что группа $\Delta$ изоморфна кокомпактной решетке в $H$.

Доказательство. Основной инструмент доказательства - теорема Керкгофа о неподвижной точке [15]. Чтобы сформулировать ее, приведем некоторые понятия из теории Тейхмюллера. Для кокомпактной решетки без кручения $\Gamma \subset$ $P S L_{2}(\mathbb{R})$ обозначим через $\mathscr{R}(\Gamma)$ множество гомоморфизмов $\varphi: \Gamma \rightarrow P S L_{2}(\mathbb{R})$ с дискретным и кокомпактным образом. Группа $P S L_{2}(\mathbb{R})$ действует на множестве $\mathscr{R}(\Gamma)$ посредством действия сопряжениями на образе. Фактор

$$
\mathbb{T}(\Gamma)=\mathscr{R}(\Gamma) / P S L_{2}(\mathbb{R})
$$

традиционно называют пространством Тейхмюллера группы Г. Обозначим через $[\varphi]$ класс в пространстве Тейхмюллера $\mathbb{T}(\Gamma)$ элемента $\varphi \in \mathscr{R}(\Gamma)$. Группа автоморфизмов $\operatorname{Aut}(\Gamma)$ действует посредством композиции на $\mathscr{R}(\Gamma)$. Это действие определяет действие группы внешних автоморфизмов $\operatorname{Out}(\Gamma)$ на $\mathbb{T}(\Gamma)$. Теорема Керкгофа утверждает, что любая конечная подгруппа в Out(Г) имеет неподвижную точку в $\mathbb{T}(\Gamma)$.

(i) Предположим, что мы находимся в ситуации, описанной в утверждении (i). Поставим в соответствие элементу $\delta \in \Delta$ автоморфизм $\varphi_{\delta}: \Gamma \rightarrow \Gamma$, где $\varphi_{\delta}(\gamma)=\delta \gamma \delta^{-1}$. Это определяет гомоморфизм $\Delta \rightarrow \operatorname{Out}(\Gamma)$. Его образ $\bar{\Delta}-$ конечная подгруппа в $\operatorname{Out}(\Gamma)$. Пусть $[\varphi] \in \mathbb{T}(\Gamma)$ - неподвижная точка для $\bar{\Gamma}$. Это означает, что для каждого $\delta \in \Delta$ существует $r_{\delta} \in P S L_{2}(\mathbb{R})$ такое, что

$$
\varphi\left(\delta \gamma \delta^{-1}\right)=r_{\delta} \gamma r_{\delta}^{-1} \quad \text { для всех } \gamma \in \Gamma .
$$

Поскольку централизатор $\varphi(\Gamma)$ в $P S L_{2}(\mathbb{C})$ тривиален, то элемент $r_{\delta}$ однозначно определяется элементом $\delta$. Это показывает, что отображение

$$
\psi: \Delta \rightarrow P S L_{2}(\mathbb{R}), \quad \psi(\delta)=r_{\delta},
$$

является гомоморфизмом групп. Легко видеть, что ядро $\psi$ равно $Z_{\Delta}(\Gamma)$. Для $\gamma \in \Gamma$ имеем $\psi(\gamma)=\varphi(\gamma)$. Отсюда мы получаем, что $\psi(\Gamma)$ - конечное расширение дискретной кокомпактной группы $\varphi(\Gamma)$. Это доказывает (i). 
(ii) Заменяя Г на одну из ее подгрупп конечного индекса, мы можем считать, что $\Gamma$ не имеет кручения и нормальна в $\Delta$. По теореме плотности Бореля центр группы $\Gamma$ тривиален, и, кроме того, $\Gamma \cap Z_{\Delta}(\Gamma)=\{1\}$. Ввиду (i), мы можем считать, что $\Delta / Z_{\Delta}(\Gamma)$ вложена в $P S L_{2}(\mathbb{R})$. Откуда следует (ii), если воспользоваться следующим очевидным инъективным гомоморфизмом:

$$
\Delta \rightarrow\left(\Delta / Z_{\Delta}(\Gamma)\right) \times(\Delta / \Gamma) \subset H=P S L_{2}(\mathbb{R}) \times(\Delta / \Gamma)
$$

Предложение доказано.

ПреДЛОЖЕНИЕ 3.4. Пусть $\Gamma=\Gamma_{1} \times \cdots \times \Gamma_{n}$ - прямое произведение конечного числа дискретных кокомпактных подгрупп $\Gamma_{i}$ в $P S L_{2}(\mathbb{R})(i=1, \ldots, n)$. Пусть $\Delta$ - конечное расширение группъ Г. Тогда $\Delta-f c f$-группа.

ДокАЗАтельство. Рассмотрим случай $n=1$. По предложению 3.3 мы имеем конечное расширение $H$ группы $P S L_{2}(\mathbb{R})$ такое, что $\Delta$ - дискретная кокомпактная подгруппа в $H$. Пусть $K \subset H$ - максимальная компактная подгруппа. Тогда фактор $H / K$ может быть отождествлен с двумерным гиперболическим пространством. Поскольку $\Delta$ действует разрывно и кокомпактно на $H / K$, наш результат следует из леммы 3.2 .

Теперь доказательство можно завершить аналогично доказательству предложения 3.2 и, воспользовавшись леммой 3.3 , получить результат для любого $n$.

Теперь мы должны вспомнить некоторые факты об индуцированных представлениях.

Пусть $G \subseteq G L_{n}(\mathbb{C})$ - линейная алгебраическая группа, $\Gamma \subseteq G$ - ее подгруппа и $\Delta \supseteq \Gamma$ - конечное расширение группы $\Gamma$ такое, что $\Gamma$ нормальна в $\Delta$. Обозначим через $H=\bar{\Gamma}$ замыкание в топологии Зариского группы $\Gamma$. Пусть $\Delta=\Gamma_{r_{1}} \cup \cdots \cup \Gamma_{r_{m}}$ - разложение $\Delta$ в объединение левых смежных классов. Предположим, что существуют рациональные гомоморфизмы $f_{1}, \ldots, f_{m}$ : $G \rightarrow G$ такие, что

$$
f_{i}(\gamma)=r_{i} \gamma r_{i}^{-1} \quad(i=1, \ldots, m)
$$

для всех $\gamma \in \Gamma$. Таким образом, имеем $f_{i}(H)=H$ для $i=1, \ldots, m$. Пусть $\psi: \Delta \rightarrow G L_{m n}(\mathbb{C})$ - вложение, согласованное с выбранными представителями смежных классов. При ограничении на $Г$ отображение $\psi$ имеет следующий вид:

$$
\psi(\gamma)=\left(\begin{array}{cccc}
r_{1} \gamma r_{1}^{-1} & 0 & \ldots & 0 \\
0 & r_{2} \gamma r_{2}^{-1} & \ldots & 0 \\
\vdots & \vdots & \ddots & \vdots \\
0 & 0 & \ldots & r_{m} \gamma r_{m}^{-1}
\end{array}\right) \quad \text { для всех } \gamma \in \Gamma .
$$

Если $r_{i} r_{j}=\gamma_{j k} r_{k}$, где $\gamma_{j k} \in \Gamma$, то в матрице $\psi\left(r_{i}\right)$ стоит $\gamma_{j k}$ на $(j, k)$-месте. В клетках, которые не определены этими условиями, стоят нули. Согласно нашим предположениям, гомоморфизм $\psi: \Gamma \rightarrow G L_{m n}(\mathbb{C})$ можно продолжить 
до рационального гомоморфизма $\Psi: H \rightarrow G L_{m n}(\mathbb{C})$, определенного формулой

$$
\Psi(h)=\left(\begin{array}{cccc}
f_{1}(h) & 0 & \cdots & 0 \\
0 & f_{2}(h) & \cdots & 0 \\
\vdots & \vdots & \ddots & \vdots \\
0 & 0 & \cdots & f_{m}(h)
\end{array}\right) \quad(h \in H) .
$$

Далее, определим

$$
I(G, \Gamma, \Delta)=\Psi(H) \cdot \psi(\Delta) .
$$

Очевидно, что $\psi(\Delta)$ нормализует $\Psi(H)$, и, следовательно, $I(G, \Gamma, \Delta)-$ подгруппа в $G L_{m n}(\mathbb{C})$. Более того, легко видеть, что $\Psi(H)$ имеет индекс $m$ в $I(G, \Gamma, \Delta)$. Следовательно, $I(G, \Gamma, \Delta)$ - алгебраическая подгруппа в $G L_{m n}(\mathbb{C})$. Имеет место следующее предложение.

ПРЕДЛОЖЕНИЕ 3.5. Пусть $G \subseteq G L_{n}(\mathbb{C})$ - линейная алгебраическая группа, определенная над $\mathbb{Q}$, и пусть $\Gamma \subseteq G(\mathbb{Z})$ - подгруппа конечного индекса. Определим $H$ как замыкание группь $\Gamma$ в топологии Зариского. Пусть $\Gamma \subseteq \Delta$ - конечное расширение такое, что группа $\Gamma$ нормальна в $\Delta$. Пусть $\Delta=\Gamma_{r_{1}} \cup \cdots \cup \Gamma_{r_{m}}-$ разложение $\Delta$ в обгединение левых смежных классов. Предположим, что существуют рачиональные гомоморфизмы $f_{1}, \ldots, f_{m}$ группы $G$, удовлетворяюшие (3.5), которые являются $\mathbb{Q}$-рачиональными при ограничении на $H$. Тогда $I(G, \Gamma, \Delta)$ - $\mathbb{Q}$-определенная линейная алгебраическая группа, а $\psi(\Delta)-\mathbb{Q}$-арифметическая подгруппа в $I(G, \Gamma, \Delta)$.

ОПРЕДЕЛЕНиЕ 3.1. Пусть $G$ - линейная алгебраическая группа, определенная над $\mathbb{Q}$. Арифметическая подгруппа $\Gamma$ группы $G$ называется $\mathbb{Q}$-арифметической, если $\Gamma \subset G(\mathbb{Q})$. Абстрактная группа $\Delta$ называется $\mathbb{Q}$-арифметической, если она изоморфна $\mathbb{Q}$-арифметической подгруппе $\mathbb{Q}$-определенной линейной алгебраической группы.

ПРЕДЛОЖЕНИЕ 3.6. Пусть Г-арифметическая группа. Тогда Г абстрактно изоморфна $\mathbb{Q}$-арифметической группе.

ДоКАЗАТЕЛЬСТвО ПРЕДЛОЖЕНИЯ 3.6. Пусть $\Gamma \subseteq G \subseteq G L_{n}(\mathbb{C})$ - арифметическая подгруппа $\mathbb{Q}$-определенной линейной алгебраической группы $G$. Пусть $\Gamma_{0}=\Gamma \cap G(\mathbb{Z})$; тогда $\Gamma_{0}-$ подгруппа конечного индекса в Г. Выберем подгруппу $\Gamma_{1}$ конечного индекса в $\Gamma_{0}$ такую, что $\Gamma_{1}$ нормальна в $\Gamma$, и определим $H$ как замыкание $\Gamma_{1}$ в топологии Зариского. Тогда $H$ - линейная алгебраическая группа, определенная над $\mathbb{Q}$. Очевидно, что выполнены условия предложения 3.5. Теперь воспользуемся вложением $\Gamma$ в $I\left(G, \Gamma_{1}, \Gamma\right)$, чтобы закончить доказательство.

ДОКАЗАТЕЛЬСТВО ТЕОРЕМЫ 3.1. Пусть Г-арифметическая группа, и пусть $\Delta$ - конечное расширение группы $\Gamma$. Мы хотим показать, что $\Delta$ является $f c f$-группой. По предложению 3.6 мы можем считать, что существует линейная алгебраическая группа $G$, определенная над $\mathbb{Q}$, такая, что $\Gamma \subseteq G(\mathbb{Q})$. Заменяя $Г$ на одну из ее подгрупп конечного индекса, мы также можем считать, что $\Gamma$ нормальна в $\Delta$. Если $\Gamma$ не плотна в $G$ в топологии Зариского, 
то заменим $G$ замыканием $\Gamma$ в топологии Зариского. Мы также можем предполагать, что группа $G$ связна.

Сначала сведем нашу проблему к случаю, когда $G$ полупроста. Пусть $R-$ разрешимый (связный) радикал группы $G$, и пусть $\pi: G \rightarrow G / R$ - соответствующий морфизм факторизации. Группа $\Gamma_{R}=\Gamma \cap R-$ арифметическая подгруппа $R$, нормальная в $\Gamma$. Подгруппа $\pi(\Gamma) \cong \Gamma / \Gamma_{R}$ группы $G$ плотна в топологии Зариского и арифметична. Пусть $R(\Gamma)$ - разрешимый радикал Г. Это наибольшая нормальная разрешимая подгруппа. Она существует, поскольку $\Gamma$ - линейная группа. Так как $\pi(R(\Gamma))$ разрешима и нормальна в $\pi(\Gamma)$, то ее замыкание в топологии Зариского, $\overline{\pi(R(\Gamma))}$, также является разрешимой и нормальной подгруппой в полупростой группе $G / R$, а следовательно, является конечной подгруппой, и $\pi(R(\Gamma))=\overline{\pi(R(\Gamma))}$. Отсюда мы делаем вывод, что индекс $\Gamma_{R}$ в $R(\Gamma)$ конечен.

Предположим теперь, что любое конечное расширение арифметической подгруппы в полупростой $\mathbb{Q}$-определенной линейной алгебраической группе является $f c f$-группой. Тогда любое конечное расширение $\Gamma / R(\Gamma) \subseteq G /(R \cdot R(\Gamma))$ является $f c f$-группой. Применяя лемму 3.4 к точной последовательности

$$
1 \rightarrow \Gamma / R(\Gamma) \rightarrow \Delta / R(\Gamma) \rightarrow \Delta / \Gamma \rightarrow 1,
$$

мы получаем, что $\Delta / R(\Gamma)-f c f$-группа. Группа $\Gamma_{R}$ является полициклической. По теореме Мальцева [16] конечное расширение полициклической группы является $f c f$-группой. Следовательно, любое конечное расширение $R(\Gamma)-$ $f c f$-группа. Применив лемму 3.4 к точной последовательности

$$
1 \rightarrow R(\Gamma) \rightarrow \Delta \rightarrow \Delta / R(\Gamma) \rightarrow 1,
$$

мы получим, что $\Delta$ является $f c f$-группой.

Покажем, что $\Delta-f c f$-группа в случае, когда $G$ полупроста. Снова можно считать, что $\Gamma$ нормальна в $\Delta$, а также что $\Gamma$ не имеет кручения. Факторизуя $G$ по (конечному) центру, мы можем считать, что группа $G$ имеет присоединенный тип. Тогда мы имеем разложение $G \cong G_{1} \times \cdots \times G_{n}$ как в лемме 3.1. А именно, $G_{1}, \ldots, G_{n}-\mathbb{Q}$-просты и ни одна из групп $G_{1}(\mathbb{R}), \ldots, G_{n}(\mathbb{R})$ не является компактной. Положим $\Gamma_{i}=G_{i} \cap \Gamma$. Группа $\Gamma_{1} \cdots \Gamma_{n}$ по лемме 3.1 снова нормальна в $\Delta$ и удовлетворяет гипотезе, наложенной на $Г$. Далее мы считаем, что $\Gamma=\Gamma_{1} \times \cdots \times \Gamma_{n}$. Перенумеруем группы $\Gamma_{1}, \ldots, \Gamma_{n}$ таким образом, что $\Gamma_{1} \cong \ldots \cong \Gamma_{k}$ и ни один из множителей $\Gamma_{k+1}, \ldots, \Gamma_{n}$ не изоморфен $\Gamma_{1}$. Положим $\Gamma_{0}=\Gamma_{1} \cdots \Gamma_{k}$ и $G_{0}=G_{1} \cdots G_{k}$. По лемме 3.1 подгруппа $\Gamma_{0}$ нормальна в $\Delta$.

Покажем сначала, что любое конечное расширение $\Delta_{0}$ группы $\Gamma_{0}$ является $f c f$-группой. Если $G_{0}(\mathbb{R})$ не имеет простого множителя, изоморфного $S L_{2}(\mathbb{R})$ или $P S L_{2}(\mathbb{R})$ с дискретной проекцией $\Gamma_{0}$ на этот множитель, то мы можем применить теорему 2.3 , что доказывает арифметичность $\Delta_{0}$. По теореме 4.2 из [1] в этом случае $\Delta_{0}$ является $f c f$-группой. Если же $G_{0}(\mathbb{R})$ имеет такой простой множитель, то существует $j \in\{1, \ldots, k\}$ такое, что $G_{j}$ - простой множитель, изоморфный $S L_{2}(\mathbb{R})$ или $P S L_{2}(\mathbb{R})$, причем проекция $\Gamma_{j}$ на этот множитель дискретна. Хорошо известно, что $\Gamma_{j}$ в этом случае изоморфна либо свободной группе конечного ранга, либо кокомпактной фуксовой группе. Эти два случая покрываются предложениями 3.2 и 4.1 . 
Используя лемму 3.4 и проведя несложную индукцию по количеству множителей $n$, мы завершим доказательство теоремы.

Пусть $F$ - конечная группа автоморфизмов арифметической группы $Г$. Тогда естественно определяется первое когомологическое множество $H^{1}(F, \Gamma)$ (см. [17]). Будет ли множество $H^{1}(F, \Gamma)$ конечным? Борель и Серр в [17] доказали конечность в ряде случаев, однако в общем случае проблема конечности оставалась открытой. С другой стороны, Борель и Серр заметили, что множество $H^{1}(F, \Gamma)$ будет конечным, если доказать, что всякое конечное расширение арифметической группы имеет конечное число классов сопряженности конечных подгрупп. Решение проблемы конечности $H^{1}(F, \Gamma)$ тогда следует из теоремы 3.1 .

ТЕОРема 3.2. Пусть $F$ - конечная группа автоморфизмов арифметической группы $Г$. Тогда множество $H^{1}(F, \Gamma)$ конечно.

\section{4. Целочисленные линейные группы с конечным числом образующих}

Поскольку любое конечное расширение арифметической группы изоморфно конечно порожденной подгруппе в $G L_{n}(\mathbb{Z})$ для некоторого $n$, автором в 1966 г. был сформулирован следующий естественный вопрос (см. [14; проблема 4]).

ПроБЛЕма 1. Пусть $\Gamma \subseteq G L_{n}(\mathbb{Z})$ - конечно порожденная подгруппа. Верно ли, что число классов сопряженности конечных подгрупп в группе Г конечно?

Эта проблема была поставлена около тридцати лет назад. Решение было найдено в работах [8], [7]. Мы приведем изложение этого решения.

Чтобы описать основную конструкцию, введем некоторые дополнительные обозначения. Если Г и $G-$ некоторые подгруппы и $\varphi: \Gamma \rightarrow G-$ групповой гомоморфизм, то определим

$$
\Gamma \times_{\varphi} \Gamma=\{(g, h) \in \Gamma \times \Gamma \mid \varphi(g)=\varphi(h)\} .
$$

Очевидно, что $\Gamma \times{ }_{\varphi} \Gamma-$ подгруппа в $\Gamma \times \Gamma$; это обратный образ в коммутативной диаграмме

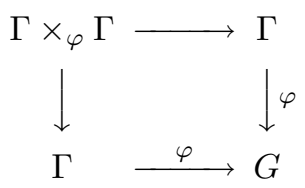

Лемма 4.1. Пусть $\Gamma$ - конечно порожденная группа, и пусть $\varphi: \Gamma \rightarrow G-$ групповой гомоморфизм такой, что ядро ч конечно порождено как нормальная подгруппа $\Gamma$. Тогда группа $\Gamma \times{ }_{\varphi} \Gamma$ конечна порождена.

ДокАЗАТЕЛЬство. Пусть $\gamma_{1}, \ldots, \gamma_{n}-$ множество образующих $\Gamma$, и пусть $r_{1}, \ldots, r_{m}-$ множество образующих ядра $\varphi$ как нормальной подгруппы в $Г$. Легко видеть, что элементы

$$
\left(\gamma_{1}, \gamma_{1}\right), \ldots,\left(\gamma_{n}, \gamma_{n}\right), \quad\left(r_{1}, 1\right), \ldots,\left(r_{m}, 1\right), \quad\left(1, r_{1}\right), \ldots,\left(1, r_{m}\right)
$$

являются образующими для $\Gamma \times{ }_{\varphi} \Gamma$. 
Лемма 4.2. Пусть $Г$ - конечно порожденная группа, и пусть $\varphi: \Gamma \rightarrow \mathbb{Z}-$ сюрвективный гомоморфизм групп. Предположим, что существует $f \in \Gamma-$ элемент конечного порядка с конечным чентрализатором $Z_{\Gamma}(f)$. Пусть элемент $z \in \Gamma$ выбран таким образом, что $\varphi(z)=1$. Тогда группа $\Gamma \times_{\varphi} \Gamma$ конечно порождена, а элементы $f_{i}=\left(z^{i} f z^{-i}, f\right)(i \in \mathbb{Z}, i \neq 0)$ попарно несопряжены в $\Gamma \times_{\varphi} \Gamma$.

ДокАЗАТЕЛЬство. Ядро $\varphi$, очевидно, конечно порождено как нормальная подгруппа в Г. Следовательно, применяя лемму 4.1, мы получаем, что группа $\Gamma \times{ }_{\varphi} \Gamma$ конечно порождена.

Предположим, что $f_{i}$ и $f_{j}$ сопряжены в $\Gamma \times{ }_{\varphi} \Gamma$. Тогда существуют $x, y \in \Gamma$ такие, что $\varphi(x)=\varphi(y)$ и

$$
\left(x z^{i} f z^{-i} x^{-1}, y f y^{-1}\right)=\left(z^{j} f z^{-j}, f\right) .
$$

Отсюда мы получаем, что $y \in Z_{\Gamma}(f)$. Поскольку группа $Z_{\Gamma}(f)$ конечна, то $0=\varphi(y)=\varphi(x)$. Из (4.1) следует, что $z^{-j} x z^{j} \in Z_{\Gamma}(f)$. Применяя $\varphi$ к последнему элементу, мы заключаем, что $i=j$.

Tеорема 4.1. Существует конечно порожденная подгруппа $\Delta \subset S L_{2}(\mathbb{Z}) \times$ $S L_{2}(\mathbb{Z})$, которая содержит бесконечное число классов сопряженности элементов порядка 4.

ДокАЗАТЕЛЬСтво. Рассмотрим следующий элемент порядка 4 в $S L_{2}(\mathbb{Z})$ :

$$
\omega=\left(\begin{array}{cc}
4 & -1 \\
17 & -4
\end{array}\right)=\left(\begin{array}{ll}
1 & 0 \\
4 & 1
\end{array}\right)\left(\begin{array}{cc}
0 & -1 \\
1 & 0
\end{array}\right)\left(\begin{array}{cc}
1 & 0 \\
-4 & 1
\end{array}\right)
$$

Непосредственным вычислением получаем, что централизатор $Z_{S L_{2}(\mathbb{Z})}(\omega)$ конечен. Заметим также, что $\omega$ содержится в

$$
\Gamma_{0}(17)=\left\{\left(\begin{array}{ll}
a & b \\
c & d
\end{array}\right) \in S L_{2}(\mathbb{Z}): 17 \mid c\right\} .
$$

Эта группа является подгруппой конечного индекса в $S L_{2}(\mathbb{Z})$. Хорошо известно (см. [18]), что существует сюръективный гомоморфизм $\varphi: \Gamma_{0}(17) \rightarrow \mathbb{Z}$. Мы можем теперь применить лемму 4.2, чтобы построить искомую подгруппу как $\Delta=\Gamma_{0}(17) \times{ }_{\varphi} \Gamma_{0}(17) \subset S L_{2}(\mathbb{Z}) \times S L_{2}(\mathbb{Z})$.

СледСтвиЕ. Существуют конечно порожденные подгруппы в $G L_{n}(\mathbb{Z})$, $n>3$, с бесконечным числом классов сопряженных конечных подгрупп.

ПредлОЖенИЕ 4.1. Пусть Г - конечно порожденная подгруппа в $G L_{2}(\mathbb{Z})$. Тогда Г - конечно представимая $f$ cf-группа.

ДокАзАтЕльство. Хорошо известно, что $G L_{2}(\mathbb{Z})$ содержит подгруппу конечного индекса, которая является конечно порожденной свободной группой ранга $\geqslant 2$. Следовательно, Г содержит либо бесконечную циклическую подгруппу, либо свободную подгруппу ранга $\geqslant 2$ в качестве подгруппы конечного индекса. В последнем случае результат следует из предложения 3.2. В первом же случае рассуждение не представляет труда. 
Аналогичный вопрос для конечно порожденных подгрупп группы $G L_{3}(\mathbb{Z})$ остается открытым.

В [19] теорема 4.1 использована для построения конечно представимой подгруппы в $G L_{6}(\mathbb{Z})$, которая содержит бесконечное число классов сопряженности элементов порядка 4.

\section{5. Проблема арифметичности для разрешимых групп}

Пусть $Г$ - арифметическая группа. По теореме 4.2 из [1] группа Г конечно представима, а из конструкции естественного индуцированного представления следует, что $\Gamma$ изоморфна подгруппе $G L_{n}(\mathbb{Z})$ для некоторого $n$.

Естественно сформулировать следующую проблему: какие конечно порожденные группы арифметичны?

Начнем с нильпотентных групп. Если $H$ - конечно порожденная нильпотентная группа, то $H$ имеет подгруппу без кручения $H_{1}$ конечного индекса. Из результатов А.И. Мальцева следует (см. [20]), что существует инъективный гомоморфизм $f: H_{1} \rightarrow U_{n}(\mathbb{Z})$ в группу $U_{n}(\mathbb{Z})$ верхних унитреугольных матриц над $\mathbb{Z}$ для которого $f\left(H_{1}\right)$ - арифметическая подгруппа в замыкании $\overline{f\left(H_{1}\right)} \subset U_{n}(\mathbb{C})$ в топологии Зариского, а также что любой автоморфизм группы $f\left(H_{1}\right)$ является ограничением рационального автоморфизма алгебраической группы $\overline{f\left(H_{1}\right)}$. По предложению 3.5 группа $H$ арифметична.

Таким образом, всякая конечно порожденная нильпотентная группа является арифметической.

Рассмотрим более широкий класс конечно порожденных разрешимых подгрупп.

Пусть $\Delta-$ конечно порожденная разрешимая группа. Если $\Delta$ арифметическая, то $\Delta$ изоморфна разрешимой подгруппе в $G L_{n}(\mathbb{Z})$ для некоторого $n$. По теореме Мальцева [16] разрешимая подгруппа в $G L_{n}(\mathbb{Z})$ является полициклической. Группа Г называется полициклической, если она обладает конечным нормальным рядом, в котором все факторы циклические. Число бесконечных циклических факторов в этих рядах является инвариантом группы $\Gamma$, который называется числом Хирша $h(\Gamma)$ группы Г. По теореме Ауслендера [21] любая полициклическая группа изоморфна подгруппе в $G L_{n}(\mathbb{Z})$ для некоторого $n$.

Следовательно, снова возникает естественный вопрос: верно ли, что полициклическая группа арифметична?

Первый контрпример был построен автором в [22]. Позднее Д. Сигал [23] построил аналогичные контрпримеры и сформулировал следующую проблему: для каких $h(\Gamma)$ полициклическая группа Г будет арифметической?

Решение проблемы арифметичности для полициклических групп было дано Груневальдом и автором в работах [5], [8].

Примеры неарифметических полициклических групп Г, построенные в [22] и [23], не имеют подгруппы конечного индекса, которая являлась бы расщепимым расширением нильпотентной группы с помощью абелевой группы. Из этого факта сразу же следует, что группа $Г$ не арифметична. Контрпримеры Груневальда-Платонова имеют принципиально новую природу: они имеют 
в качестве подгрупп любого сколь угодно большого индекса расщепимые расширения абелевых подгрупп с помощью абелевых подгрупп. Но, оказывается, намного сложнее показать, что эти группы не арифметические.

Tеорема 5.1. Пусть $h$ - натуральное число такое, что $h \geqslant 4$. Тогда существуют неарифметические поличиклические группы с числом Хирша $h$, которые являются расщепимыми расширениями $\mathbb{Z}^{h-1}$ с помощъю бесконечной ицклической группъ.

Поскольку любая конечно порожденная нильпотентная группа арифметична, эти примеры являются минимальными.

Теорема 5.2. Минимальное число Хириа неарифметической полициклической группъ равно 4.

Напомним, что подгруппой Фиттинга группы $Г$ называется подгруппа, порожденная всеми нормальными нильпотентными подгруппами $Г$. Если $Г-$ линейная группа, то подгруппа Фиттинга $\mathscr{F}(\Gamma)$ является нильпотентной нормальной подгруппой.

Линейная алгебраическая группа $G$ действует посредством сопряжения на своем унипотентном радикале $U(G)$. Мы называем $G$ редуцированной (или группой с сильным унипотентным радикалом), если ядро этого действия содержится в $U(G)$.

ЛЕмма 5.1. Пусть $G$-редуцированная, определенная над $\mathbb{Q}$ линейная алгебраическая группа, являющаяся конечным расширением разрешимой группь u $\Gamma \subseteq G$ - арифметическая подгруппа. Тогда $\mathscr{F}(\Gamma)=U(G, \Gamma)$.

ДоказАтеЛЬство. Очевидно, что $U(G, \Gamma)$ содержится в $\mathscr{F}(\Gamma)$. Пусть $\gamma-$ элемент из группы $\mathscr{F}(\Gamma)$, положим $A=U(G, \Gamma) \cdot\langle\gamma\rangle$, и пусть $\bar{A}$ - замыкание группы $A$ в топологии Зариского. Тогда $\bar{A}$ - нильпотентная линейная алгебраическая группа. Пусть $\gamma=\gamma_{u} \cdot \gamma_{s}$ - разложение Жордана элемента $\gamma$, где $\gamma_{u}$ унипотентен, а $\gamma_{s}$ полупрост и они коммутируют между собой. Мы также имеем $\gamma_{u}, \gamma_{s} \in \bar{A}$. Так как $U(G, \Gamma) \subseteq A$, то $U(G) \subseteq \bar{A}$. Следовательно, группа $U(G) \cdot\left\langle\gamma_{s}\right\rangle$ нильпотентна, откуда $\gamma_{s}=1$, поскольку $G$ редуцирована. Значит, $\gamma$ унипотентен. По теореме из [24] имеет место разложение $G=U(G) \cdot S$, где $S-$ расширение тора с помощью конечной группы. Пусть $\gamma=\gamma_{1} \times \gamma_{2}$, где $\gamma_{1} \in U(G)$ и $\gamma_{2} \in S$. Поскольку $\gamma$ и $\gamma_{1}$ унипотентны, имеем $\gamma_{2}=1$, откуда следует наш результат.

Следующая теорема играет важную роль при решении проблемы арифметичности для разрешимых групп.

Теорема 5.3 (см. [8; теорема 3.4]). Пусть Г - арифметическая подгруппа разрешимой $\mathbb{Q}$-определенной линейной алгебраической группы $G$. Подгруппа Г изоморфна плотной в топологии Зариского арифметической подгруппе группь $H(\mathbb{Q})$, где $H$ - редуиированная разрешимая $\mathbb{Q}$-определенная линейная алгебрачческая группа $H$, тогда и толъко тогда, когда подгруппа Фиттинга группы Г не имеет кручения.

В качестве немедленного следствия из теоремы 5.3 получаем следующее утверждение. 
СлЕДСтвиЕ. Пусть Г - арифметическая подгруппа $\mathbb{Q}$-определенной линейной алгебраической группы $G$ с разрешимой связной компонентой $G^{\circ}$. Тогда существуют подгруппа $\Gamma_{0}$ группы $\Gamma$ конечного индекса и редуцированная разрешимая $\mathbb{Q}$-определенная линейная алгебрачческая группа $H$ такие, что $\Gamma_{0}$ изоморфна плотной в топологии Зариского арифметической подгруппе групnъь $H(\mathbb{Q})$.

Мы начнем с арифметической конструкции некоторых полициклических групп, дающей примеры неарифметических групп, которые нам понадобятся при доказательстве теоремы 5.1.

Пусть $K$ - числовое поле и $\mathscr{O}_{K}$ - его кольцо целых. Предположим, что $K$ имеет $r_{1}$ вещественных и $2 r_{2}$ комплексных вложений. Тогда $K$ имеет степень $n=r_{1}+2 r_{2}$ над $\mathbb{Q}$. Обозначим через $\mathscr{O}_{K}^{*}$ группу единиц кольца $\mathscr{O}_{K}$. По теореме Дирихле о единицах имеем изоморфизм $\mathscr{O}_{K}^{*} \cong C \times \mathbb{Z}^{r}$, где $C$ - конечная циклическая группа и $r_{K}=r_{1}+r_{2}-1$. Обозначим $\mathscr{O}_{K}^{+}$аддитивную группу $\mathscr{O}_{K}$. Группа $\mathscr{O}_{K}^{*}$ действует умножением $\mathscr{O}_{K}^{+}$. Пусть $\mathscr{O}_{K}^{+} \rtimes \mathscr{O}_{K}^{*}-$ соответствующее расщепимое расширение. Пусть теперь $G_{\mathrm{a}}, G_{\mathrm{m}}$ - аддитивная и мультипликативная группы, рассматриваемые как линейные алгебраические группы над $K$. Тогда $G_{\mathrm{m}}$ действует умножениями на $G_{\mathrm{a}}$, обозначим через $G_{\mathrm{a}} \rtimes G_{\mathrm{m}}$ соответствующее расщепимое расширение. Пусть $G_{K}=R_{K / \mathbb{Q}}\left(G_{\mathrm{a}} \rtimes G_{\mathrm{m}}\right)=$ $R_{K / \mathbb{Q}}\left(G_{\mathrm{a}}\right) \rtimes R_{K / \mathbb{Q}}\left(G_{\mathrm{m}}\right)$ - функтор ограничения основного поля рассматриваемой группы до поля $\mathbb{Q}$. Тогда $G_{K}$ - редуцированная разрешимая линейная алгебраическая группа, определенная над $\mathbb{Q}$, а также имеют место естественные отождествления:

$$
G_{K}(\mathbb{Q})=K^{+} \rtimes K^{*}, \quad G_{K}(\mathbb{Z})=\mathscr{O}_{K}^{+} \rtimes \mathscr{O}_{K}^{*} .
$$

Пусть $A \subseteq \mathscr{O}_{K}^{*}-$ некоторая подгруппа. Положим

$$
\Gamma_{K}(A)=\mathscr{O}_{K}^{+} \rtimes A \subseteq \mathscr{O}_{K}^{+} \rtimes \mathscr{O}_{K}^{*} .
$$

Группа $\Gamma_{k}(A)$ - полициклическая, а ее подгруппа Фиттинга $\mathscr{F}\left(\Gamma_{K}(A)\right)$ равна аддитивной группе $\mathscr{O}_{K}$, которая естественно вложена в $\Gamma_{K}(A)$. Следовательно, $\mathscr{F}\left(\Gamma_{K}(A)\right) \cong \mathbb{Z}^{n}$. Формулируемое ниже предложение характеризует подгруппы $A \subseteq \mathscr{O}_{K}^{*}$, для которых $\Gamma_{K}(A)$ - арифметическая группа.

ПРеДЛОЖЕНИЕ 5.1. Пусть $K$ - алгебраическое числовое поле степени $n$ над $\mathbb{Q}$. Тогда группа $\Gamma_{K}(A)$ является арифметической, если и только если существует $\mathbb{Q}$-определенная алгебраическая подгруппа $S \subseteq R_{K / \mathbb{Q}}\left(G_{\mathrm{m}}\right)$ такая, что А содержится в качестве плотной в топологии Зариского арифметической подгруппы в $S$.

ДокАзАтЕльство. Пусть $S \subseteq R_{K / \mathbb{Q}}\left(G_{\mathrm{m}}\right)$ - $\mathbb{Q}$-определенная алгебраическая подгруппа. Если $A \subseteq S$ соизмерима с $S(\mathbb{Z})$, то группа $\Gamma_{K}(A)$, очевидно, является арифметической подгруппой $\mathbb{Q}$-определенной линейной алгебраической группы $R_{K / \mathbb{Q}}\left(G_{\mathrm{a}}\right) \rtimes S$.

Предположим теперь, что группа $\Gamma_{K}(A)$, построенная выше, является арифметической. Применяя теорему 5.3 , мы находим редуцированную разрешимую $\mathbb{Q}$-определенную линейную алгебраическую группу $H$ такую, что существует 
изоморфизм $\psi: \Gamma_{K}(A) \rightarrow \Gamma$, где $\Gamma$ - плотная в топологии Зариского арифметическая подгруппа $H(\mathbb{Q})$. Отождествим $\mathscr{O}_{K}^{+}$и $A$ с их вложениями в $\Gamma_{K}(A)$. По лемме 5.1 подгруппа Фиттинга группы $\Gamma$ равна $\Gamma \cap U(H)$. Мы получаем, что $\psi\left(\mathscr{O}_{K}^{+}\right)=\Gamma \cap U(H)$. Предположим с этого момента, что $A \neq\{1\}$.

Легко видеть, что $A$ - максимальная коммутативная подгруппа $\Gamma_{K}(A)$. Пусть $S_{0}$ - замыкание в топологии Зариского группы $\psi(A)$ в $H$. Это $\mathbb{Q}$-определенная коммутативная алгебраическая подгруппа в $H$. Следовательно, $\Gamma \cap S_{0}$ - арифметическая подгруппа в $S_{0}$. Поскольку $\psi(A)$ - максимальная коммутативная подгруппа $\Gamma$, имеем $\Gamma \cap S_{0}=\psi(A)$, и, значит, $\psi(A)$ - плотная по Зарискому арифметическая подгруппа $S_{0}(\mathbb{Q})$.

По лемме 5.1 и приведенным выше замечаниям мы находим, что алгебра Ли $\mathfrak{U}(H)(\mathbb{Q})$ - абелева размерности $n$ над $\mathbb{Q}$ и $\exp ^{-1}(\Gamma \cap U(H))=\exp ^{-1}\left(\psi\left(\mathscr{O}_{K}^{+}\right)\right)$абелева подгруппа $\mathfrak{U}(H)(\mathbb{Q})$, которая содержит $\mathbb{Q}$-базис этого пространства. Выберем $\mathbb{Q}$-базис $\mathscr{B}$ в аддитивной группе $\mathscr{O}_{K}^{+}$поля $K$, рассматриваемого как $\mathbb{Q}$-векторное пространство. Это дает нам рациональное $\mathbb{Q}$-определенное инъективное представление $\Lambda: R_{K / \mathbb{Q}}\left(G_{\mathrm{m}}\right) \rightarrow G L_{n}(\mathbb{C})$. Для элемента $k \in K^{*}$ его образ $\Lambda(k)$ является матрицей, соответствующей оператору умножения на $k$. Заметим, что $\Lambda$ дает рациональный определенный над $\mathbb{Q}$ изоморфизм между $R_{K / \mathbb{Q}}\left(G_{\mathrm{m}}\right)$ и замыканием в топологии Зариского образа $\Lambda\left(K^{*}\right) \subseteq G L_{n}(\mathbb{C})$. Гомоморфизм $\exp ^{-1} \circ \psi: \mathscr{O}_{K}^{+} \rightarrow \mathfrak{U}(H)(\mathbb{Q})$ может быть продолжен до $\mathbb{Q}$-линейного изоморфизма $\Theta: K \rightarrow \mathfrak{U}(H)(\mathbb{Q})$. Выбирая базис $\Theta(\mathscr{B})$ для $\mathfrak{U}(H)(\mathbb{Q})$, мы получаем рациональное $\mathbb{Q}$-определенное представление $\alpha_{H}: H \rightarrow G L_{n}(\mathbb{C})$. Для представлений $\Lambda, \alpha_{H}$, очевидно, выполнено равенство

$$
\Theta^{-1} \circ \alpha_{H}(\psi(a)) \circ \Theta=\Lambda(a)
$$

для всех $a \in A$. Положим $S=\Theta^{-1} \alpha_{H}\left(S_{0}\right) \Theta$. Подгруппа $S \subset G L_{n}(\mathbb{C})$ определена над $\mathbb{Q}$. Образ $\psi(A)$ в $S$ плотен в топологии Зариского и по теореме плотности Бореля также является арифметической подгруппой в $S$. Воспользовавшись (5.1), мы видим, что $\Lambda(A)$ - плотная по Зарискому арифметическая подгруппа в $S$. Это доказывает предложение.

Напомним некоторые важные сведения из теории коммутативных $d$-групп. Линейная коммутативная группа $G$ называется $d$-группой, если $G$ состоит из полупростых элементов. Пусть $\overline{\mathbb{Q}}$ - алгебраическое замыкание $\mathbb{Q}$ в $\mathbb{C}$, а $\mathbb{G}$ - его группа Галуа. Для $\mathbb{Q}$-определенной коммутативной $d$-группы $S$ пусть $X(S)$ группа (鸟-определенных) характеров $S$.

Если $\varphi: S_{1} \rightarrow S_{2}$ - определенный над $\mathbb{Q}$ рациональный гомоморфизм между двумя коммутативными $\mathbb{Q}$-определенными $d$-группами $S_{1}, S_{2}$, обозначим $X(\varphi): X\left(S_{2}\right) \rightarrow X\left(S_{1}\right)$ для соответствующего гомоморфизма между модулями характеров. Это дает контравариантную функториальную эквивалентность между категорией $\mathbb{Q}$-определенных $d$-групп и категорией непрерывных $\mathbb{G}$-модулей, которые конечно порождены как $\mathbb{Z}$-модули. Мы будем писать $S_{1} \sim S_{2}$ в случае, когда $S_{1}$ и $S_{2}$ изогенны. Тогда $S_{1} \sim S_{2}$, если и только если $\mathbb{Q} \otimes_{\mathbb{Z}} X\left(S_{1}\right)$ и $\mathbb{Q} \otimes_{\mathbb{Z}} X\left(S_{2}\right)$ изоморфны как $\mathbb{G}$-модули. Для коммутативной $d$-группы $S$ пусть $\operatorname{dim}(S)$ - ее размерность как алгебраической группы. Имеем $\operatorname{dim}(S)=$ $\operatorname{dim}_{\mathbb{Q}}\left(\mathbb{Q} \otimes_{\mathbb{Z}} X(S)\right)$. Если $L \subseteq \mathbb{C}-$ некоторое подполе, то $S$ будем называть 
$L$-расщепимой, если все элементы $X(S)$ определены над $L$. Легко видеть, что существует минимальное поле расщепления $L_{S}$ для $S$. Это такое подполе, что $L_{S} \subseteq \overline{\mathbb{Q}}$, если $S$ является $L_{S}$-расщепимой, и $L_{S} \subseteq L$ для любого другого поля $L \subseteq \mathbb{C}$, для которого $S$ является $L$-расщепимой.

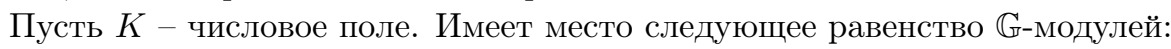

$$
X\left(R_{K / \mathbb{Q}}\left(G_{\mathrm{m}}\right)\right)=\mathbb{Z}[\mathbb{G} / \mathbb{U}(K)],
$$

где $\mathbb{U}(K) \subseteq \mathbb{G}$ - открытая подгруппа конечного индекса в $\mathbb{G}$, стабилизирующая элементы поля $K$, а $\mathbb{Z}[\mathbb{G} / \mathbb{U}(K)]$ - соответствующий премутационный модуль. При этом $d$-группа $R_{K / \mathbb{Q}}\left(G_{\mathrm{m}}\right)$ расщепима над замыканием Галуа $\widehat{K}$ поля $K$ в $\overline{\mathbb{Q}}$. Норменное отображение $N_{K / \mathbb{Q}}: K \rightarrow \mathbb{Q}$ определяет характер группы $R_{K / \mathbb{Q}}\left(G_{\mathrm{m}}\right)$, определенный над $\mathbb{Q}$. Пусть $R_{K / \mathbb{Q}}^{1}\left(G_{\mathrm{m}}\right)$ - его ядро. Модуль характеров группы $R_{K / \mathbb{Q}}^{1}\left(G_{\mathrm{m}}\right)$ изоморфен $\mathbb{Z}[\mathbb{G} / \mathbb{U}(K)] / \mathbb{Z}$. Имеем

$$
R_{K / \mathbb{Q}}^{1}\left(G_{\mathrm{m}}\right)(\mathbb{Z})=\mathscr{O}_{K}^{1}
$$

где $\mathscr{O}_{K}^{1}$ обозначает группу единиц с нормой 1 в кольце $\mathscr{O}_{K}$.

Далее мы покажем, что предложение 5.1 может быть использовано для конструкции неарифметических полициклических групп.

ПРЕДЛОЖЕНИЕ 5.2. Пусть $K$ - вполне вещественное числовое поле степени $[K: \mathbb{Q}]=n \geqslant 3$. Тогда:

(i) с точностью до соизмеримости существует только конечное число бесконечных циклических подгрупп $A \subseteq \mathscr{O}_{K}^{*}$ maкuх, что группа $\Gamma_{K}(A)$ арифметична;

(ii) если $K$ - расширение Галуа $\mathbb{Q} u[K: \mathbb{Q}]$ нечетно, то группа $\Gamma_{K}(A)$ не является арифметической ни для какой бесконечной ииклической подгруппы $A \subseteq \mathscr{O}_{K}^{*}$

ДокАЗАТЕЛЬСтво. Чтобы доказать предложение, достаточно рассмотреть случай бесконечных циклических подгрупп $A \subseteq \mathscr{O}_{K}^{1}$, для которых группа $\Gamma_{K}(A)$ арифметична. Положим $T=R_{K / \mathbb{Q}}\left(G_{\mathrm{m}}\right)$, и пусть $S \subseteq T$ - определенная над $\mathbb{Q}$ алгебраическая подгруппа такая, что $A$ арифметична и плотна в топологии Зариского в $S$. Поскольку $K$ вполне вещественно, то $d$-группа $T$ является $\mathbb{R}$-расщепимой. На самом деле мы имеем $\mathbb{R}$-определенный рациональный изоморфизм $T(\mathbb{R}) \cong\left(\mathbb{R}^{*}\right)^{n-1}$. Более того, $T(\mathbb{Z})$ - дискретная подгруппа в $T(\mathbb{R})$ и (по теореме Дирихле о единицах) группа $T(\mathbb{R}) / T(\mathbb{Z})$ компактна. Следовательно, группа $S$ является $\mathbb{R}$-расщепимой и $\operatorname{dim}(S)=1$. Пусть $L_{S}-$ поле расщепления $S$. Поскольку $\mathbb{Q} \otimes_{\mathbb{Z}} X(S)$ одномерно, имеем $\left[L_{S}: \mathbb{Q}\right] \leqslant 2$. Так как $S(\mathbb{Z})$ бесконечна, то $S$ не может быть $\mathbb{Q}$-расщепимой. Следовательно, $\left[L_{S}: \mathbb{Q}\right]=2$. Пусть $\widehat{K}$ - замыкание Галуа поля $K$. Поскольку группа $S$ является $K$-расщепимой, мы получаем $L_{S} \subseteq K$. Это доказывает утверждение (ii) нашего предложения. Чтобы доказать (i), рассмотрим $S_{1}=R_{L_{S} / \mathbb{Q}}\left(G_{\mathrm{m}}\right) \subseteq T$. Поскольку всякое неприводимое одномерное представление $\mathbb{G}$ встречается в $\mathbb{Q} \otimes_{\mathbb{Z}} X(T)$ по крайней мере один раз, мы получаем, что $S=S_{1}$ и $A$ соизмеримы с $S_{1}(\mathbb{Z})$. Наконец, заметим, что поле $\widehat{K}$ имеет лишь конечное число квадратичных подполей. Предложение доказано. 
Предложение 5.1 позволяет построить намного больше примеров неарифметических групп $\Gamma_{K}(A)$, чем те, что встречаются в предложении 5.2. Для общих (не вполне вещественных) числовых полей $K$ ситуация является более сложной. Это показывает следующее предложение.

ПРЕДЛОЖЕНИЕ 5.3. Пусть $K$ - расширение Галуа степени 6 над $\mathbb{Q}$ с группой Галуа, изоморфной симметрической группе $S_{3}$. Пусть $K_{0}$ - его единственное квадратичное подполе. Предположим, что $K_{0}$ - мнимое квадратичное поле. Тогда $\Gamma_{K}(A)$ - арифметическая группа для каждой подгруппы $A \subseteq \mathscr{O}_{K}^{*}$.

ДокАЗАТЕЛЬство. Поскольку $K$ - чисто мнимое квадратичное поле, группа $\mathscr{O}_{K}^{*}$ имеет подгруппу конечного индекса, изоморфную $\mathbb{Z}^{2}$. Из разложения группового кольца $\mathbb{Q}\left[S_{3}\right]$ на неприводимые двусторонние идеалы мы находим, что

$$
R_{K / \mathbb{Q}}\left(G_{\mathrm{m}}\right) \sim T_{0} \times T_{1} \times T_{2},
$$

где группа $T_{0}$ изогенна $R_{K_{0} / \mathbb{Q}}\left(G_{\mathrm{m}}\right)$, а группы $T_{1}$ и $T_{2}$ - торы, определенные над $\mathbb{Q}$, которые изоморфны над $\mathbb{Q}$. Из этого следует, что оба тора $T_{1}(\mathbb{Z})$ и $T_{2}(\mathbb{Z})$ содержат бесконечную циклическую подгруппу конечного индекса. Очевидно, что группа $T_{0}(\mathbb{Z})$ конечна. Поскольку $T_{1} \times T_{2}$ содержат определенные над $\mathbb{Q}$ одномерные торы вида $\left\{\left(t_{1}^{a}, t_{2}^{b}\right) \mid t_{1} \in T_{1}, t_{2} \in T_{2}\right\}$ для $a, b \in \mathbb{N}$, наш результат следует из предложения 5.1 .

ДокаЗАТЕЛЬСтво теоремы 5.1. Пусть дано $h \in \mathbb{N}, h \geqslant 4$. По теореме Дирихле о простых числах в арифметической прогрессии, существует простое число $p$ такое, что $(p-1) / 2$ делится на $h-1$. Легко видеть, что циклотомическое расширение степени $p-1$ поля $\mathbb{Q}$ содержит подполе, которое является вполне вещественным степени $h-1$ над $\mathbb{Q}$. Теперь мы можем применить предложение 5.2. Теорема доказана.

Хорошо известно, что существуют вполне вещественные числовые поля $K$ степени $n \geqslant 3$ над $\mathbb{Q}$ такие, что группа Галуа замыкания Галуа поля $K$ изоморфна знакопеременной группе $A_{n}$. Теорема 5.1 может быть получена (так же как и выше) из следующего предложения.

ПреДЛОЖЕНИЕ 5.4. Пусть $K$ - вполне вещественное числовое поле степени $n \geqslant 3$ над $\mathbb{Q}$. Предположим, что группа Галуа замыкания Галуа поля $K$ изоморфна знакопеременной группе $A_{n}$. Тогда $\Gamma_{K}(A)$ не является арифметической ни для какой бесконечной ииклической подгруппы $A \subseteq \mathscr{O}_{K}^{*}$.

ДокАЗАТЕльство. Предположим, что $A \subseteq \mathscr{O}_{K}^{*}-$ бесконечная циклическая подгруппа такая, что $\Gamma_{K}(A)$ - арифметическая группа. По предложению 5.1 найдется $\mathbb{Q}$-определенный тор $S \subseteq R_{K / \mathbb{Q}}\left(G_{\mathrm{m}}\right)$ такой, что группа $A$ соизмерима с $S(\mathbb{Z})$. Аналогично доказательству предложения 5.2 можно показать, что тор $S$ одномерен и поле расщепления $L_{S}$ тора $S$ имеет степень 2 над $\mathbb{Q}$. Кроме того, $L_{S}$ содержится в замыкании Галуа поля $K$. Что противоречит нашим предположениям. Предложение доказано.

ДОКАЗАТЕЛЬСТВО ТЕОРЕМЫ 5.2. Пусть Г - полициклическая группа, у которой инвариант Хирша не превосходит 3. Мы хотим показать, что группа Г 
арифметична. Хорошо известно, что Г содержит подгруппу без кручения конечного индекса $\Gamma_{0}$ такую, что $\Gamma_{0} / \mathscr{F}\left(\Gamma_{0}\right)$ также без кручения. Воспользовавшись следствием из теоремы 5.3, мы видим, что для доказательства теоремы достаточно показать, что $\Gamma_{0}$ арифметична. Разберем возможные варианты строения группы Фиттинга.

Случай 1: $h\left(\mathscr{F}\left(\Gamma_{0}\right)\right)=3$. В этом случае $\Gamma_{0}$ нильпотентна. Любая конечно порожденная нильпотентная подгруппа арифметична согласно результату Мальцева.

Случай 2: $h\left(\mathscr{F}\left(\Gamma_{0}\right)\right)=2$. В этом случае $\mathscr{F}\left(\Gamma_{0}\right)$ абелева и, как легко видеть, $\mathscr{F}\left(\Gamma_{0}\right) \cong \mathbb{Z}^{2}$. Согласно выбору $\Gamma_{0}$, имеем $\Gamma_{0} / \mathscr{F}\left(\Gamma_{0}\right) \cong \mathbb{Z}$. Рассмотрим $\sigma \in \Gamma_{0}$ такой, что его образ порождает фактор $\Gamma_{0} / \mathscr{F}\left(\Gamma_{0}\right)$. Положим $T=\langle\sigma\rangle$. Тогда имеем изоморфизм $\Gamma_{0}=\mathscr{F}\left(\Gamma_{0}\right) \cdot T \cong \mathscr{F}\left(\Gamma_{0}\right) \rtimes T$. Действие сопряжениями элементом $\sigma$ на $\mathscr{F}\left(\Gamma_{0}\right)$ дает матрицу $A \in G L_{2}(\mathbb{Z})$. Положим $T_{1}=\langle A\rangle$. Элементы $T_{1}$ действуют линейно на $\mathbb{Z}^{2}$. Рассмотрим расщепимое расширение $\Gamma_{1}=\mathbb{Z}^{2} \rtimes T_{1}$. Группы $\Gamma_{0}$ и $\Gamma_{1}$ изоморфны. Пусть $K-\mathbb{Q}$-алгебра, порожденная $A$ в кольце матриц размера $2 \times 2$. По теореме Дирихле о единицах $K$ - вещественное квадратичное расширение $\mathbb{Q}$. Рассмотрим действие мультипликативной группы $G_{\mathrm{m}}$ на аддитивной группе $G_{\text {a }}$ умножениями и образуем соответствующее расщепимое расширение $G_{\mathrm{a}} \rtimes G_{\mathrm{m}}$. Легко видеть, что $\Gamma_{1}$ изоморфна арифметической подгруппе группы ограничения скаляров $R_{K / \mathbb{Q}}\left(G_{\mathrm{a}} \times G_{\mathrm{m}}\right)$ в $G_{\mathrm{a}} \times G_{\mathrm{m}}$.

Легко видеть, что случаи $h\left(\mathscr{F}\left(\Gamma_{0}\right)\right)=0,1$ невозможны. Теорема доказана.

Пусть Г - полициклическая группа. По теореме Ауслендера группа автоморфизмов $\operatorname{Aut}(\Gamma)$ изоморфна конечно представимой подгруппе из $G L_{n}(Z)$ для подходящего $n$. Поэтому естественно возникает вопрос об арифметичности группы $\operatorname{Aut}(\Gamma)$.

Пусть $\Gamma_{K}(A)$ - построенные в предложениях 5.2 и 5.4 полициклические группы, не являющиеся арифметическими. Можно показать, что группа автоморфизмов $\operatorname{Aut}\left(\Gamma_{K}(A)\right)$ не является арифметической.

На основе результатов работ [7] и [8] в [25] построены примеры разрешимых арифметических групп $\Gamma$, для которых $\operatorname{Aut}(\Gamma)$ не является арифметической. Для группы внешних автоморфизмов $\operatorname{Out}(\Gamma)=\operatorname{Aut}(\Gamma) / \operatorname{Inn}(\Gamma)$ в [25] получен следующий общий результат.

ТеОремА 5.4. Пусть Г - конечное расширение поличиклической группь. Тогда группа Out(Г) арифметична.

\section{6. Решетки в группах Ли}

Пусть $G$ - вещественная группа Ли с конечным числом связных компонент. Дискретная подгруппа $\Gamma \subset G$ называется решеткой в $G$, если однородное пространство $G / \Gamma$ имеет конечную $G$-инвариантную меру. Решетка $\Gamma$ в $G$ называется кокомпактной, если пространство $G / \Gamma$ компактно.

Абстрактная группа называется решеткой, если она изоморфна решетке в вещественной группе Ли с конечным числом связных компонент.

Оказывается, что для решеток справедливы результаты, аналогичные результатам разделов 2,3 (см. [18]). 
Если группа $G$ является полупростой, то большинство решеток, но не все, являются арифметическими группами. В свою очередь, если $Г$ - арифметическая подгруппа $\mathbb{Q}$-определенной полупростой алгебраической группы $G$, то $\Gamma \cap G(\mathbb{R})$ является решеткой в $G(\mathbb{R})$.

Мы будем называть группу $\Delta$ конечным расширением группы $\Gamma$, если $\Delta$ содержит подгруппу конечного индекса, изоморфную Г.

Пусть теперь $\Delta$ - конечное расширение решетки Г. Будет ли Г решеткой? Контрпример, построенный в теореме 2.1 для арифметических групп, является контрпримером и для решеток.

Сейчас мы дадим полное решение проблемы конечных расширений для решеток в $P S L_{2}(\mathbb{R})$.

Теорема 6.1. Пустъ Г является решеткой в $P S L_{2}(\mathbb{R})$. Тогда справедливъ следующие утверждения:

1) если $\Gamma$ кокомпактна в $P S L_{2}(\mathbb{R})$ u $\Delta$ - конечное расширение $\Gamma$, то $\Delta$ является решеткой;

2) если Г не является кокомпактной, то существует конечное расширение $\Delta$ группы $Г$, не являющееся решеткой.

Для доказательства второй части теоремы 6.1 нам понадобится следующее утверждение, доказательство которого фактически содержится в доказательстве теоремы 2.1 .

ПРЕДЛОЖЕНИЕ 6.1. Пусть $\Delta$ - конечное расширение решетки $\Gamma \subset P S L_{2}(\mathbb{R})$. Предположим, что $\Delta$ является решеткой в вещественной группе Ли. Тогда $G=H \cdot K$, где $H$ и $K$ являются замкнутыми нормальными подгруппами группъ $G$, причем $K$ - компактная подгруппа, $H$ - конечное расширение группь $P S L_{2}(\mathbb{R})$ и пересечение $H \cap K$ является конечной центральной подгруппой. Если $\Delta$ не имеет нетривиальных конечных нормальных подгрупп, то $\Delta$ изоморфна решетке в $P G L_{2}(\mathbb{R})$.

Пусть теперь $\Gamma$ - решетка в $P S L_{2}(\mathbb{R})$, не являющаяся кокомпактной. Хорошо известно (см. [26]), что Г имеет следующее строение: Г является свободным произведением $l \in \mathbb{N} \cup\{0\}$ бесконечных циклических групп и $n \in \mathbb{N} \cup\{0\}$ конечных циклических групп, т. е.

$$
\Gamma=\left\langle h_{1}, \ldots, h_{l}, e_{1}, \ldots, e_{n} \mid e_{1}^{m_{1}}=\cdots=e_{n}^{m_{n}}=1\right\rangle \cong \underbrace{\mathbb{Z} * \cdots * \mathbb{Z}}_{l \text { раз }} * \mathbb{Z}_{m_{1}} * \cdots * \mathbb{Z}_{m_{n}},
$$

где $\mathbb{Z}_{m}$ обозначает конечную циклическую группу порядка $m$. Условие, что группа $\Gamma$, определенная в (6.1), изоморфна не кокомпактной решетке в $P S L_{2}(\mathbb{R})$, является следующим:

$$
l-1+\sum_{l=1}^{n}\left(1-\frac{1}{m_{i}}\right)>0 .
$$

ДокАЗАТЕЛЬСтво тЕОРЕмы 6.1. Утверждение 1) теоремы 6.1 следует из предложения 3.3. Доказательство утверждения 2) проводится путем рассмотрения различных случаев. 
Случай 1: $l=2, n=0$. Здесь $\Gamma=\left\langle h_{1}, h_{2}\right\rangle$ - свободная группа с двумя образующими. Рассмотрим следующие три автоморфизма группы $\Gamma$ :

$$
\sigma_{1}\left(h_{1}\right)=h_{1}^{-1}, \quad \sigma_{2}\left(h_{2}\right)=h_{2}^{-1}, \quad \sigma_{3}\left(h_{1}\right)=h_{2}, \quad \sigma_{3}\left(h_{2}\right)=h_{1},
$$

здесь мы считаем, что $\sigma_{i}$ действуют тривиально на образующей, если действие не указано. Пусть $A$ - подгруппа группы автоморфизмов $\Gamma$, порожденная $\sigma_{1}$, $\sigma_{2}, \sigma_{3}$, положим $\Delta=\Gamma \rtimes A$. Группа $A$ изоморфна группе диэдра порядка 8 , тем самым индекс $\Gamma$ в $\Delta$ равен 8 . Группа $\Delta$ не является решеткой. Чтобы это увидеть, заметим, что у $\Delta$ нет нетривиальных конечных нормальных подгрупп. Мы получаем из предложения 6.1 , что если $\Delta$ - решетка, то она также решетка в $P G L_{2}(\mathbb{R})$. Мы отождествим $\Delta$ с ее образом в $P G L_{2}(\mathbb{R})$ и положим $\Delta^{\circ}=\Delta \cap P S L_{2}(\mathbb{R})$. В силу вышесказанного $\Delta^{\circ}$ не является кокомпактной решеткой в $P S L_{2}(\mathbb{R})$ и имеет место разложение (6.1). Отсюда по теореме о подгруппах свободных произведений [7] мы получаем, что любая абелева подгруппа группы $\Delta^{\circ}$ является циклической. Следовательно, $\Delta^{\circ}$ имеет индекс 2 в $\Delta$. Мы также находим, что $\Delta^{\circ} \cap A$ - циклическая порядка 4 и, следовательно, порождается $\sigma_{3} \sigma_{1}$. Отсюда также следует, что $\sigma_{2} \sigma_{1}=\left(\sigma_{3} \sigma_{1}\right)^{2}$ лежит в $\Delta^{\circ}$, но $\sigma_{1}$ не лежит в $\Delta^{\circ}$. Заметим, что $\sigma_{2} \sigma_{1}$ - инволюция, централизуемая $\sigma_{1}$. Простое вычисление показывает, что централизатор в $P G L_{2}(\mathbb{R})$ любой инволюции из $P S L_{2}(\mathbb{R})$ содержится в $P S L_{2}(\mathbb{R})$. Это заканчивает доказательство того, что $\Delta$ не является решеткой.

Из структуры $\Delta$ легко видеть, что существует единственная подгруппа индекса 2 в $\Delta$, которая изоморфна решетке в $P S L_{2}(\mathbb{R})$. Эта подгруппа изоморфна $\mathbb{Z}_{2} * \mathbb{Z}_{4}$

Случай $2: l \geqslant 2, l+n \geqslant 3$. В этом случае у решетки $Г$ должны быть образующие $h_{1}, h_{2}$ и по крайней мере еще одна образующая $f$ (равная $h_{3}$ или $e_{1}$ ) из разложения (6.1). Пусть $A$ - подгруппа группы автоморфизмов $\Gamma$, порожденная $\sigma_{1}, \sigma_{2}, \sigma_{3}$, приведенными в (6.2) (с предположением, что $\sigma_{i}$ действуют тривиально на образующей, если действие не указано). Снова положим $\Delta=\Gamma \rtimes A$. Тогда $\Delta$ содержит $\Gamma$ в качестве подгруппы индекса 8 и $\Delta$ не является решеткой. Чтобы это увидеть, снова заметим, что у $\Delta$ нет нетривиальных конечных нормальных подгрупп. Из предложения 6.1 мы получаем, что если $\Delta$ - решетка, то она также решетка в $P G L_{2}(\mathbb{R})$. Пусть $B$ - замыкание в топологии Зариского подгруппы $T=\left\langle h_{2}, f\right\rangle$ в $P G L_{2}(\mathbb{R})$. Поскольку $T$ не является разрешимой, группа $B$ содержит $P G L_{2}(\mathbb{R})$. Элемент $\sigma_{1}$ централизует $T$, следовательно, он централизует и $B$. Приходим к противоречию.

Случай 3: $l=1$. В этом случае имеем $n \geqslant 1$. Рассмотрим случай $n=1$. Пусть $\Gamma_{1}$ - ядро очевидного гомоморфизма $\Gamma \rightarrow \mathbb{Z}_{m_{1}}$. Эта группа свободна порождена элементами

$$
h_{1}, \quad h_{2}=e_{1} h_{1} e_{1}^{-1}, \quad \ldots, \quad h_{m_{1}}=e_{1}^{m_{1}-1} h_{1} e_{1}^{1-m_{1}} .
$$

Симметрическая группа $S_{m_{1}}$ очевидным образом вкладывается в группу автоморфизмов Г посредством перестановки образующих из (6.3). Далее, мы можем вложить $\mathbb{Z}_{2}^{m_{1}}$ в качестве подгруппы $A_{0}$ группы автоморфизмов $\Gamma_{1}$, полагая, что элемент $\varepsilon=\left(\varepsilon_{1}, \ldots, \varepsilon_{m_{1}}\right) \quad\left(\varepsilon_{i}= \pm 1\right)$ действует по формуле $\varepsilon\left(h_{i}\right)=h_{i}^{\varepsilon_{i}}$. 
Пусть $A$ - подгруппа группы автоморфизмов $\Gamma$, порожденная группами $A_{0}$ и $S_{m_{1}}$. Группа $A$ изоморфна сплетению $A_{0} 2 S_{m_{1}}$ и имеет порядок $2^{m_{1}} m_{1}$ !. Положим $\Delta=\Gamma_{1} \rtimes A$, очевидно, что Г изоморфна подгруппе индекса $2^{m_{1}}\left(m_{1}-1\right)$ ! в $\Delta$. Группа $\Delta$ не является решеткой. Для $m_{1}=2$ эта группа изоморфна группе из случая $l=2, n=0$, также названной $\Delta$, и мы уже знаем, что в этом случае $\Delta$ не решетка. Для $m_{1} \geqslant 3$ мы заметим, что $\Delta$ не имеет нетривиальных конечных нормальных подгрупп и, следовательно, вкладывается в $P G L_{2}(\mathbb{R})$. Но все конечные подгруппы этой группы либо циклические, либо группы диэдра. Если $n>1$, то аналогично случаю $l \geqslant 2, l+n \geqslant 3$ мы имеем расширение $\Delta$ группы $\Gamma$, которое не является решеткой.

Случай $4: l=0$. Сначала рассмотрим случай $n=2$. В этом случае либо $m_{1}$, либо $m_{2}$ строго больше, чем 2 . Если $m_{1}=2, m_{2}=3$, обозначим через $\Gamma_{1}$ ядро очевидного гомоморфизма $\Gamma \rightarrow \mathbb{Z}_{2}$. Эта группа изоморфна группе $\mathbb{Z}_{3} * \mathbb{Z}_{3}$, которая, аналогично случаю $l=2, n=0$, содержит группу диэдра $\mathbb{D}_{4}$ порядка 8 в качестве подгруппы в группе автоморфизмов. Положим $\Delta=\Gamma_{1} \rtimes \mathbb{D}_{4}$. Тогда $\Delta$ содержит $\Gamma$ в качестве подгруппы индекса 4 . Рассуждая далее аналогично случаю $l=2, n=0$, мы получаем, что группа $\Delta$ не является решеткой. Если $m_{1}=2, m_{2}=4$, то, как мы заметили в случае $l=2, n=0$, существует расширение индекса 2 , которое не является решеткой. Если $m_{1}=3, m_{2}=3$ или $m_{1}=3, m_{2}=4$, то обозначим через $\Gamma_{1}$ ядро очевидного гомоморфизма $\Gamma \rightarrow \mathbb{Z}_{m_{2}}$ и найдем подходящее сплетение в группе автоморфизмов группы $\Gamma_{1}$ (см. случай $l=1$ ). В этом случае расщепимое расширение $\Delta=\Gamma_{1} \rtimes A$ содержит $\Gamma$ в качестве подгруппы конечного индекса и не является решеткой. Во всех остальных случаях мы можем считать, что $m_{2} \geqslant m_{1}$. Снова обозначим через $\Gamma_{1}$ ядро гомоморфизма $\Gamma \rightarrow \mathbb{Z}_{m_{2}}$. Эта группа изоморфна прямому произведению $m_{2}$ копий $\mathbb{Z}_{m_{1}}$. Следовательно, группа автоморфизмов группы $\Gamma_{1}$ содержит группу перестановок $S_{m_{2}}$. Группа $\Delta=\Gamma_{1} \rtimes S_{m_{2}}$ содержит $Г$ как подгруппу индекса $\left(m_{2}-1\right)$ !. Повторяя рассуждения, приведенные ранее, снова получаем, что группа $\Delta$ не является решеткой. Случаи $n \geqslant 3$ разбираются аналогично. Пусть $m_{n} \geqslant m_{i}$ для $i=1, \ldots, n$, и пусть $\Gamma_{1}-$ ядро очевидного гомоморфизма $\Gamma \rightarrow \mathbb{Z}_{m_{n}}$. Эта группа изоморфна свободному произведению конечных циклических групп, и ее группа автоморфизмов содержит подходящую симметрическую подгруппу $S$. Группа $\Delta:=\Gamma_{1} \rtimes S$ содержит $\Gamma$ как подгруппу, и, следовательно, $\Delta-$ снова не решетка. Теорема доказана.

Из теоремы 6.1 немедленно следует теорема 2.2.

Утверждение 1) теоремы 6.1 допускает следующее максимальное обобщение.

Теорема 6.2. Пусть Г - кокомпактная решетка в вещественной группе Ли G с конечным числом связных компонент. Тогда всякое конечное расширение группы Г является решеткой.

Доказательство теоремы 6.2 использует теорему 6.1, результаты о жесткости для решеток в полупростых вещественных группах Ли (см. [27]), а также некоторые свойства жесткости для дискретных подгрупп вещественных групп Ли с радикалом (см. [7], [3]). 


\section{Список литературы}

[1] В.П. Платонов, А.С. Рапинчук, Алгебраические группь и теория чисел, Наука, M., 1991, ISBN: 5-02-014191-7, 656 с.; англ. пер.: V. Platonov, A. Rapinchuk, Algebraic groups and number theory, Pure Appl. Math., Academic Press, Boston, MA, 1994, ISBN: 0-12-558180-7, 614 pp.

[2] В.П. Платонов, “Арифметическая теория алгебраических групп”, УМН, 37:3 (1982), 3-54; англ. пер.: V. P. Platonov, "The arithmetic theory of algebraic groups", Russian Math. Surveys, 37:3 (1982), 1-62.

[3] F. Grunewald, V. Platonov, "New properties of lattices in Lie groups", C. R. Math. Acad. Sci. Paris, 338:4 (2004), 271-276.

[4] F. Grunewald, V. Platonov, "On finite extensions of arithmetic groups", C. R. Acad. Sci. Paris Sér. I Math., 325:11 (1997), 1153-1158.

[5] F. Grunewald, V. Platonov, "Non-arithmetic polycyclic groups", C. R. Acad. Sci. Paris Sér. I Math., 326:12 (1998), 1359-1364.

[6] F. Grunewald, V. Platonov, "Rigidity and automorphism groups of solvable arithmetic groups", C. R. Acad. Sci. Paris Sér. I Math., 327:5 (1998), 427-432.

[7] F. Grunewald, V. Platonov, "Rigidity results for groups with radical cohomology of finite groups and arithmeticity problems", Duke Math. J., 100:2 (1999), 321-358.

[8] F. Grunewald, V. Platonov, "Solvable arithmetic groups and arithmeticity problems", Internat. J. Math., 10:3 (1999), 327-366.

[9] G. Prasad, "Discrete subgroups isomorphic to lattices in Lie groups", Amer. J. Math., 98:4 (1976), 853-863.

[10] M. R. Bridson, "Geodesics and curvature in metric simplicial complexes", Group theory from a geometrical viewpoint (Trieste, 1990), World Sci. Publ., River Edge, NJ, 1991, 373-463.

[11] J.-P. Serre, Trees, Springer-Verlag, Berlin-New York, 1980, ISBN: 3-540-10103-9, $142 \mathrm{pp}$.

[12] W. Dicks, M. J. Dunwoody, Groups acting on graphs, Cambridge Stud. Adv. Math., 17, Cambridge Univ. Press, Cambridge, 1989, ISBN: 0-521-23033-0, 283 pp.

[13] M. Bridson, A. Haefliger, Metric spaces of non-positive curvature, Grundlehren Math. Wiss., 319, Springer-Verlag, Berlin, 1999, ISBN: 3-540-64324-9, 643 pp.

[14] В. П. Платонов, "Теория алгебраических линейных групп и периодические группы", Изв. АН СССР. Сер. матем., 30:3 (1966), 573-620; англ. пер.: V. P. Platonov, "Theory of algebraic linear groups and periodic groups", Amer. Math. Soc. Transl. Ser. 2, 69 (1968), 61-110.

[15] S. P. Kerckhoff, "The Nielsen realization problem", Ann. of Math. (2), 117:2 (1983), 235-265.

[16] А. И. Мальцев, “О некоторых классах бесконечных разрешимых групп”, Матем. сб., 28:3 (1951), 567-588.

[17] A. Borel, J.-P. Serre, "Théorèmes de finitude en cohomologie galoisienne", Comment. Math. Helv., 39 (1964), 111-164.

[18] H. Rademacher, "Über die Erzeugenden von Kongruenzuntergruppen der Modulgruppe", Abh. Math. Sem. Univ. Hamburg, 7:1 (1929), 134-148.

[19] M. R. Bridson, "Finiteness properties for subgroups of $G L(n, \mathbb{Z})$ ", Math. Ann., 317:4 (2000), 629-633.

[20] А. И. Мальцев, "Об одном классе однородных пространств", Изв. АН СССР. Сер. матем., 13:1 (1949), 9-32; англ. пер.: A. I. Mal'tsev, "On a class of homogeneous spaces", Amer. Math. Soc. Transl., 39 (1951), 33 pp. 
[21] L. Auslander, "On a problem of Philip Hall", Ann. of Math. (2), 86:1 (1967), 112-116.

[22] В. П. Платонов, "О конгруэнц-проблеме для разрешимых целочисленных групп”, Докл. АН БССР, 15:2 (1971), 869-872.

[23] D. Segal, Polycyclic groups, Cambridge Tracts in Math., 82, Cambridge Univ. Press, Cambridge, 1983, ISBN: 0-521-24146-4, xiv+289 pp.

[24] В. П. Платонов, "Разрешимые алгебраические группы", Докл. АН CCCP, 151:1 (1963), 48-51; англ. пер.: V. P. Platonov, "Solvable algebraic groups", Soviet Math. Dokl., 4 (1963), 924-927.

[25] O. Baues, F. Grunewald, "Automorphism groups of polycyclic-by-finite groups and arithmetic groups", Publ. Math. Inst. Hautes Études Sci., 104:1 (2006), 213-268.

[26] L. Greenberg, "Finiteness theorems for Fuchsian and Kleinian groups", Discrete groups and automorphic functions (Cambridge, 1975), Academic Press, London, 1977, 199-257.

[27] G.A. Margulis, Discrete subgroups of semisimple Lie groups, Ergeb. Math. Grenzgeb. (3), 17, Springer-Verlag, Berlin, 1991, ISBN: 3-540-12179-X, x+388 pp.

В. П. Платонов (V.P. Platonov)

Научно-исследовательский институт

системных исследований РАН

E-mail: platonov@niisi.ras.ru
Поступила в редакцию

08.06.2010 